TRANSACTIONS OF THE

AMERICAN MATHEMATICAL SOCIETY

Volume 348, Number 4, April 1996

\title{
WHITEHEAD TEST MODULES
}

\author{
JAN TRLIFAJ
}

\begin{abstract}
A (right $R$-) module $N$ is said to be a Whitehead test module for projectivity (shortly: a p-test module) provided for each module $M$, $\operatorname{Ext}_{R}(M, N)=0$ implies $M$ is projective. Dually, i-test modules are defined. For example, $\mathbb{Z}$ is a p-test abelian group iff each Whitehead group is free. Our first main result says that if $R$ is a right hereditary non-right perfect ring, then the existence of p-test modules is independent of ZFC $+\mathrm{GCH}$. On the other hand, for any ring $R$, there is a proper class of i-test modules. Dually, there is a proper class of p-test modules over any right perfect ring.

A non-semisimple ring $R$ is said to be fully saturated ( $\kappa$-saturated) provided that all non-projective ( $\leq \kappa$-generated non-projective) modules are i-test. We show that classification of saturated rings can be reduced to the indecomposable ones. Indecomposable 1-saturated rings fall into two classes: type I, where all simple modules are isomorphic, and type II, the others. Our second main result gives a complete characterization of rings of type II as certain generalized upper triangular matrix rings, $G T(1, n, p, S, T)$. The four parameters involved here are skew-fields $S$ and $T$, and natural numbers $n, p$. For rings of type I, we have several partial results: e.g. using a generalization of Bongartz Lemma, we show that it is consistent that each fully saturated ring of type $I$ is a full matrix ring over a local quasi-Frobenius ring. In several recent papers, our results have been applied to Tilting Theory and to the Theory of *-modules.
\end{abstract}

In modern algebra, the structure of rings, $R$, is studied by means of properties of corresponding module categories, Mod-R. In most cases, it is not possible to characterize $M o d-R$ fully. Nevertheless, there are important subclasses of Mod- $R$ that can be treated in detail and that shed light on the whole of Mod-R. Among the prominent ones are the classes of all projective and all injective modules.

Recall that a module $M$ is said to be projective (injective) provided that the functor $\operatorname{Hom}_{R}(M,-)\left(\operatorname{Hom}_{R}(-, M)\right)$ preserves short exact sequences. There is also a universal algebraic aspect: each module is a factor module of a projective module, and a submodule of an injective module. So a possible strategy to investigate Mod$R$ consists in describing all injective modules, and for each injective module, $I$, all its submodules. The first step is usually relatively easy, but the second may be quite hard. For example, using this strategy for abelian groups, one meets serious difficulties already for $I=\mathbb{Q} \oplus \mathbb{Q}$ (see e.g. [E, Theorem 2]).

Received by the editors March 17, 1995.

1991 Mathematics Subject Classification. Primary 16E30; Secondary 03E35, 20K35.

Key words and phrases. Whitehead test module, injective module, projective module, uniformization, weak diamond, generalized upper triangular matrix rings.

Research supported by grant GAUK-44. 
There are two basic results on testing injectivity and projectivity. First, Baer's Criterion, saying that injectivity can be tested using only cyclic (singular) modules. Then Kaplansky's Theorem, saying that any projective module is a direct sum of countably generated (projective) modules. This theorem enables one to test projectivity of certain modules by their $\Gamma$-invariants (see [EM, IV, §1]).

In the present paper, we suggest another way to test projectivity and injectivity. It consists in evaluations of appropriate extension groups Ext. Of course, a module $M$ is projective iff $\operatorname{Ext}_{R}(M, N)=0$ for all modules $N \in M o d-R$. Similarly, $N$ is injective iff $\operatorname{Ext}_{R}(M, N)=0$ for all modules $M \in M o d-R$.

When we are testing for projectivity or injectivity, we need not check all the groups $\operatorname{Ext}_{R}(M, N)$. In fact, the evaluation of a single group is enough. For each module $M$, there exist modules $K$ and $L$ such that $M$ is projective (injective) iff $\operatorname{Ext}_{R}(M, K)=0\left(\operatorname{Ext}_{R}(L, M)=0\right)$-see Lemma 1.1 below. The problem is that the modules $K$ and $L$ depend on $M$, and they can be quite big provided $M$ is such. We would like to get rid of this dependence, deciding the projectivity or injectivity of $M$ by calculating a single Ext group using a fixed module $N$. This leads to the following basic definition:

Definition. Let $R$ be a ring and $N$ be a module.

(i) $N$ is said to be a Whitehead test module for projectivity (or a p-test module) provided that for each $M \in M o d-R, \operatorname{Ext}_{R}(M, N)=0$ implies $M$ is projective.

The class of all p-test modules is called the $p$-test class and denoted by $\mathcal{P} \mathcal{T}$.

(ii) $N$ is said to be a Whitehead test module for injectivity (or an i-test module) provided that for each $M \in M o d-R, \operatorname{Ext}_{R}(N, M)=0$ implies $M$ is injective. The class of all i-test modules is called the $i$-test class and denoted by $\mathcal{I} \mathcal{T}$.

Note that it can be quite hard to decide whether a particular module is p-test or not. For example, if $R=\mathbb{Z}$, then the question "Is $\mathbb{Z}$ a p-test $\mathbb{Z}$-module?" is exactly the well-known Whitehead problem. Thanks to a series of deep results of Shelah, notably [S1], [S2], [S3] and [S4], this problem is known to be undecidable in ZFC + GCH (see also [EM, Ch.XII]). Recently, Eklof and Shelah have given a full answer to this problem, by identifying its combinatorial equivalent [ES2].

In Sections 1-3 of this paper, we deal with existence of p-test and i-test modules. We show that the i-test class is a proper class for an arbitrary ring (Corollary 1.3). Dually, the p-test class is a proper class for any right perfect ring (Corollary 1.5). The more difficult question of existence of p-test modules over non-right perfect rings cannot be answered using only algebraic methods. Here, the set-theoretic methods developed by Shelah for abelian groups turn out to be very useful. In Section 2, the consistency of non-existence is proved using a uniformization principle due to Shelah (Theorem 2.5). On the other hand, in Section 3, a generalized weak diamond principle is applied to prove consistency of existence of a proper class of p-test modules over any right hereditary non-right perfect ring (Theorem 3.13).

In Sections 4-6, we study rings possessing many test modules. A non-semisimple ring $R$ is said to be fully saturated ( $\kappa$-saturated) provided that all non-projective ( $\leq \kappa$-generated non-projective) modules are i-test (Definition 4.1$)$. The fully saturated rings have already been studied, under the term "right Ext-rings", in [T1]. The results of Sections 4-6 provide substantial refinements and further progress in the structure theory instigated by [T1]. First, we show that the classification of saturated rings can be reduced to the indecomposable ones (Theorem 4.9). Then we 
divide indecomposable 1-saturated rings into two classes: type I, where all simple modules are isomorphic, and type II, the others (Definition 4.10). In Section 5, we provide a complete characterization of rings of type II as certain generalized upper triangular matrix rings, $G T(1, n, p, S, T)$ (Theorem 5.14). Finally, in Section 6, we deal with rings of type I, proving e.g. that it is consistent that each fully saturated ring of type I is a full matrix ring over a local quasi-Frobenius ring (Theorem 6.16).

The results of this paper have applications to Tilting Theory and to the Theory of $*$-modules. In [CT1, §3], [T3, §2] and [T4], they were used for a classification of almost $*$-modules. In [CT2, $\S 2]$, they were applied to an investigation of tilting torsion theories, generalizing results of Assem [As], Smalø [Sm] et al.

We use the following notation and conventions: All rings are associative and with unit. Let $R$ and $S$ be rings. Then $R \boxplus S$ denotes the ring direct product of $R$ and $S$. Further, Mod- $R$ denotes the category of all (unitary right $R$-)modules. Homomorphisms of module categories are written as acting on the opposite side from scalars. If $M$ is a module, then $\operatorname{gen}(M)$ denotes the minimal cardinality of a generating set of $M$. Further, $\operatorname{Soc}(M), \operatorname{Rad}(M)$ and $\operatorname{Sing}(M)$ denote the left socle, Jacobson radical and singular submodule of $M$, respectively. The injective hull of $M$ is denoted by $I(M)$. The $i$-th derived functor of the $H_{o m}$ functor is denoted by $E x t_{R}^{i}$, and $E x t_{R}=E x t_{R}^{1}$. For basic properties of these notions, and for further standard terminology, we refer to $[\mathrm{AF}],[\mathrm{CaEi}]$ and $[\mathrm{EM}]$.

\section{WhITEHEAD TEST MODULES FOR PROJECTIVITY AND INJECTIVITY}

We start with the simple fact that projectivity and injectivity of a given module can be tested by checking a single Ext group:

Lemma 1.1. Let $R$ be a ring.

(i) Let $M \in M o d-R$ and

$$
0 \rightarrow K \stackrel{\nu}{\rightarrow} P \rightarrow M \rightarrow 0
$$

be a short exact sequence in $M o d-R$ such that $P$ is projective. Then $M$ is projective iff $\operatorname{Ext}_{R}(M, K)=0$.

(ii) Let $N \in \operatorname{Mod}-R$ and

$$
0 \rightarrow N \rightarrow I \rightarrow L \rightarrow 0
$$

be a short exact sequence in Mod-R such that $I$ is injective. Then $N$ is injective iff $\operatorname{Ext}_{R}(L, N)=0$.

Proof. (i) Using the definition of Ext by Hom groups of the given projective presentation of $M$, we get $\operatorname{Ext}_{R}(M, K) \simeq \operatorname{Hom}_{R}(K, K) / \operatorname{Im}\left(\operatorname{Hom}_{R}(\nu, K)\right)$. Assume $\operatorname{Ext}_{R}(M, K)=0$. Then $i d_{K}=\pi \nu$, for some $\pi \in \operatorname{Hom}_{R}(P, K)$. So $\operatorname{Ker}(\pi)$ is a summand of $P$ and $\operatorname{Ker}(\pi) \simeq P / \operatorname{Im}(\nu) \simeq M$.

(ii) Dual to (i).

Using Baer's Criterion, it is easy to see that i-test modules exist over an arbitrary ring: 
Proposition 1.2. Let $R$ be a ring. Let $\mathcal{E}$ be the set of all proper essential right ideals of $R$. Put $M=\bigoplus \sum_{I \in \mathcal{E}} R / I$. Then $M$ is an i-test module.

Proof. Assume $\operatorname{Ext}_{R}(M, N)=0$. Let $J$ be a left ideal of $R$ and $\phi \in \operatorname{Hom}_{R}(J, N)$. There exist $J \subseteq I \in \mathcal{E}$ and $\bar{\phi} \in \operatorname{Hom}_{R}(I, N)$ such that $\bar{\phi}\lceil J=\phi$. By the premise, $\operatorname{Ext}_{R}(R / I, N)=0$. Since the sequence

$$
0 \rightarrow \operatorname{Hom}_{R}(R / I, N) \rightarrow \operatorname{Hom}_{R}(R, N) \rightarrow \operatorname{Hom}_{R}(I, N) \rightarrow \operatorname{Ext}_{R}(R / I, N)=0
$$

is exact, there is some $\varphi \in \operatorname{Hom}_{R}(R, N)$ such that $\varphi \uparrow I=\bar{\phi}$. By Baer's Criterion, $N$ is injective.

Of course, any module possessing a summand isomorphic to an i-test module is likewise i-test. This implies

Corollary 1.3. For any ring $R$, there is a proper class of $i$-test modules.

Clearly, each (projective) module is i-test iff the $\operatorname{ring} R$ is semisimple. Denote by $\mathcal{P}$ the class of all projective modules. Then $\mathcal{I} \mathcal{T} \subseteq \operatorname{Mod}-R \backslash \mathcal{P}$ provided $R$ is not semisimple. Though $\mathcal{I} \mathcal{I}$ is a proper class, almost never does $\mathcal{I} \mathcal{T}=\operatorname{Mod}-R \backslash \mathcal{P}$. We shall consider this problem in detail in $§ 4-6$.

Proposition 1.4. Let $R$ be a right perfect ring. Denote by $\mathcal{M}$ the set of all maximal right ideals of $R$. Put $N=\bigoplus \sum_{I \in \mathcal{M}} R / I$. Then $N$ is a p-test module.

Proof. Assume $\operatorname{Ext}_{R}(M, N)=0$ and $M$ is non-projective. Let

$$
0 \rightarrow K \rightarrow P \rightarrow M \rightarrow 0
$$

be a projective cover of $M$, i.e. a short exact sequence with $P$ projective and $K$ a superfluous submodule of $P$. By the premise, $K \neq 0$ and $K$ has a maximal submodule, $L$. Then $K / L$ is isomorphic to a summand of $N$, and $\operatorname{Ext}_{R}(M, K / L)=$ 0 . Let $\pi \in \operatorname{Hom}_{R}(K, K / L)$ be the projection. By the definition of Ext using Hom groups of the projective cover of $M$, there is a $\phi \in \operatorname{Hom}_{R}(P, K / L)$ such that $\phi \uparrow K=\pi$. Then $\operatorname{Ker}(\phi)$ is a maximal submodule of $P$ and $K \subseteq \operatorname{Rad}(P) \subseteq$ $\operatorname{Ker}(\phi) \subset P$. Thus, $\pi=\phi \uparrow K=0$, a contradiction.

Note that the proof of 1.4 can easily be dualized to obtain another (longer) proof of 1.2 .

Corollary 1.5. Let $R$ be a right perfect ring. Then there is a proper class of p-test modules.

Clearly, each (injective) module is p-test iff the $\operatorname{ring} R$ is semisimple. Denote by $\mathcal{I}$ the class of all injective modules. Then $\mathcal{P} \mathcal{T} \subseteq M$ od- $R \backslash \mathcal{I}$ provided $R$ is not semisimple. Though $\mathcal{P} \mathcal{T}$ is a proper class over any right perfect ring, almost never does $\mathcal{P} \mathcal{T}=\operatorname{Mod}-R \backslash \mathcal{I}$. Also this problem will be considered in detail in $\S 4-6$.

Finally, we introduce two important notions often used in the sequel:

Definition 1.6. Let $\kappa$ be an infinite regular cardinal. Let $R$ be a ring and $M$ be a module.

(i) $M$ is $\kappa$-projective provided that each submodule of $M$ generated by $<\kappa$ elements is projective.

(ii) A sequence $\mathcal{M}=\left(M_{\alpha} ; \alpha<\kappa\right)$ of submodules of $M$ is said to be a $\kappa$-filtration of $M$ provided that $\operatorname{gen}\left(M_{\alpha}\right)<\kappa$ for all $\alpha<\kappa, \mathcal{M}$ is a chain (i.e. $M_{\alpha} \subseteq M_{\alpha+1}$ for all $\alpha<\kappa), \mathcal{M}$ is continuous (i.e. $M_{\alpha}=\bigcup_{\beta<\alpha} M_{\beta}$ for all limit ordinals $\alpha<\kappa$ ), and $M=\bigcup_{\alpha<\kappa} M_{\alpha}$. 


\section{The USE OF UNIFORMIZATION PRINCIPLES}

In this and in the subsequent section, we show how methods and results of infinite combinatorics are used to answer the question of existence of p-test modules over non-right perfect rings. As in the particular case of $R=\mathbb{Z}$, the answer turns out to be independent of ZFC. In fact, many of the methods developed by Shelah for abelian groups carry over to modules over non-perfect rings (cf. [EM], [ES1], [T2] et al.)

In this section, we show that there is no p-test module over any non-right perfect ring, assuming Shelah's uniformization principle UP (Theorem 2.5). Moreover, there exist non-right perfect rings over which there are no finitely generated p-test modules in ZFC (Example 2.6).

We start with several notions from infinite combinatorics:

Definition 2.1. Let $R$ be a non-right perfect ring. By Bass' Theorem $\mathrm{P}$ ([AF, Theorem 28.4]), there exist elements $a_{i} \in R, i<\aleph_{0}$, such that $\left(R a_{i} \ldots a_{0} ; i<\aleph_{0}\right)$ is a strictly decreasing chain of principal left ideals of $R$. Let $\kappa$ be an infinite cardinal and $E$ be a subset of $\kappa^{+}$such that $E \subseteq\left\{\alpha<\kappa^{+} ; \operatorname{cf}(\alpha)=\aleph_{0}\right\}$. Let $\left(n_{\nu} ; \nu \in E\right)$ be a ladder system i.e. for each $\nu \in E$, let $\left(n_{\nu}(i) ; i<\aleph_{0}\right)$ be a strictly increasing sequence of non-limit ordinals less that $\nu$ such that $\sup _{i<\aleph_{0}} n_{\nu}(i)=\nu$.

Let $\left(R_{\alpha} ; \alpha<\kappa^{+}\right)$be a system of free modules defined as follows: $R_{\alpha}=R$ provided $\alpha \in \kappa^{+} \backslash E$, and $R_{\alpha}=R^{\left(\aleph_{0}\right)}$ provided $\alpha \in E$. For $\alpha \in \kappa^{+} \backslash E$, denote by $1_{\alpha}$ the canonical generator of $R_{\alpha}$, and for $\alpha \in E$ let $\left\{1_{\alpha, i} \mid i<\aleph_{0}\right\}$ be the canonical basis of $R_{\alpha}$. Note that by Bass' Lemma ([AF, Lemmas 28.1 and 28.2]), for every $\nu \in E$, the module

$$
S_{\nu}=\sum_{i<\aleph_{0}}\left(1_{\nu, i}-1_{\nu, i+1} \cdot a_{i}\right) R
$$

is a free submodule of $R_{\nu}$ such that $R_{\nu} / S_{\nu}$ is not projective. Put

$$
P=\bigoplus \sum_{\alpha<\kappa^{+}} R_{\alpha}, Q=\sum_{\alpha \in E} Q_{\alpha}, \quad \text { and } \quad Q_{\alpha}=\sum_{i<\aleph_{0}} g_{\alpha i} R
$$

for all $\alpha \in E$, where $g_{\alpha i}=\left(1_{n_{\alpha}(i)}-1_{\alpha, i}+1_{\alpha, i+1} \cdot a_{i}\right) \in P$, for all $\alpha \in E$ and $i<\aleph_{0}$. Finally, put $M=P / Q \in M o d-R$.

Recall that $E$ is a stationary subset of a cardinal $\lambda$ provided $E$ has a non-empty intersection with any closed and cofinal subset of $\lambda$.

Lemma 2.2. If $E$ is a stationary subset of $\kappa^{+}$, then $\operatorname{proj} \cdot \operatorname{dim}(M)=1$.

Proof. Put $M_{0}=0$ and, for each $0<\alpha<\kappa^{+}, M_{\alpha}=\left(\bigoplus \sum_{\beta<\alpha} R_{\beta}+Q\right) / Q$. Then $\left(M_{\alpha} ; \alpha<\kappa^{+}\right)$is a $\kappa^{+}$-filtration of $M$. Clearly, $Q=\bigoplus \sum_{\alpha \in E} Q_{\alpha}, Q_{\alpha}=$ $\bigoplus \sum_{i<\aleph_{0}} g_{\alpha i} R$ for all $\alpha \in E$, and $\operatorname{Ann}\left(g_{\alpha i}\right)=0$ for all $\alpha \in E$ and $i<\aleph_{0}$. In particular, both $P$ and $Q$ are free modules, so $\operatorname{proj} \operatorname{dim}(M) \leq 1$. Proving indirectly, assume $\operatorname{proj.} \operatorname{dim}(M)=0$, i.e. $M$ is projective. By Kaplansky's Theorem, there exist modules $\left(P_{\alpha} ; \alpha<\kappa^{+}\right)$such that gen $\left(P_{\alpha}\right) \leq \aleph_{0}$ for all $\alpha<\kappa^{+}$and $M=$ $\bigoplus \sum_{\alpha<\kappa^{+}} P_{\alpha}$. Put $N_{0}=0$ and, for each $0<\alpha<\kappa^{+}, N_{\alpha}=\bigoplus \sum_{\beta<\alpha} P_{\beta}$. Clearly, $\left(N_{\alpha} ; \alpha<\kappa^{+}\right)$is a $\kappa^{+}$-filtration of $M$. Since the set $C=\left\{\alpha<\kappa^{+} ; M_{\alpha}=N_{\alpha}\right\}$ is closed and cofinal in $\kappa^{+}$, there exists $\nu \in E \cap C$. Of course, $D=C \cap\left\{\alpha<\kappa^{+} ; \nu<\alpha\right\}$ is also closed and cofinal in $\kappa^{+}$, whence there is some $\mu \in E \cap D$. Then $X=N_{\mu} / N_{\nu}$ 
is a projective module. On the other hand, put $Y=\left(\bigoplus \sum_{\nu<\alpha<\mu} R_{\alpha}+Q\right) / Q$. Then $X=M_{\mu} / M_{\nu}=M_{\nu+1} / M_{\nu}+\left(Y+M_{\nu}\right) / M_{\nu}$. By 2.1, $Y \cap M_{\nu+1} \subseteq M_{\nu}$, whence $M_{\nu+1} / M_{\nu} \simeq R_{\nu} / S_{\nu}$ is a non-projective summand of $X$, a contradiction.

The following (meta-) lemma is proved by forcing e.g. in [ES1, §2]:

Lemma 2.3. Let $\kappa$ be a cardinal such that $\mathrm{cf}(\kappa)=\aleph_{0}$. Consider the following assertion:

$\mathrm{UP}_{\kappa}$ : "there exist a stationary subset $E$ of $\kappa^{+}$satisfying $E \subseteq\left\{\alpha<\kappa^{+} ; \operatorname{cf}(\alpha)=\aleph_{0}\right\}$ and a ladder system $\left(n_{\nu} ; \nu \in E\right)$ such that for each cardinal $\lambda<\kappa$ and each sequence $\left(h_{\nu} ; \nu \in E\right)$ of mappings from $\aleph_{0}$ to $\lambda$ there is a mapping $f: \kappa^{+} \rightarrow \lambda$ such that $\forall \nu \in E \exists j<\aleph_{0} \forall j<i<\aleph_{0}: f\left(n_{\nu}(i)\right)=h_{\nu}(i) "$.

Denote by UP the assertion " $U P_{\kappa}$ holds for every uncountable cardinal $\kappa$ such that $\mathrm{cf}(\kappa)=\aleph_{0} "$. Then $U P$ is consistent with $Z F C+G C H$.

The next lemma is proved in [T2] (cf. [ES1, Theorem 1.7]):

Lemma 2.4. Let $\kappa$ be a cardinal such that $\mathrm{cf}(\kappa)=\aleph_{0}$. Assume $\mathrm{UP}_{\kappa}$ holds. Let $M=P / Q$ be the module corresponding to the $E$ and $\left(n_{\nu}(i) ; \nu \in E\right)$ from $\mathrm{UP}_{\kappa}$ by 2.1. Then $\operatorname{Ext}(M, N)=0$ for all $N \in \operatorname{Mod}-R$ such that $\operatorname{card}(N)<\kappa$.

Now, we easily obtain the consistency result (cf. [ES1, Corollary 2.2] and [T2]):

Theorem 2.5. The assertion "There is no p-test module over any non-right perfect ring" is consistent with $Z F C+G C H$.

Proof. By 2.3, we assume $U P$. Let $N$ be a module. Let $\kappa$ be a cardinal such that $\operatorname{cf}(\kappa)=\aleph_{0}$ and $\kappa>\operatorname{card}(N)$. By 2.2 and 2.4, there is a non-projective module $M$ such that $\operatorname{Ext}_{R}(M, N)=0$. Hence, $N$ is not a p-test module.

The following example shows (in ZFC) that there exist non-right perfect rings without finitely generated p-test modules:

Example 2.6. Let $R$ be a right self-injective nonright perfect ring (e.g. let $R$ be the ring of all linear transformations of an infinite dimensional right linear space over a skew-field). Then no finitely generated module is a p-test module.

Proof. Let $a_{i}, i<\aleph_{0}$, be as in 2.1. Let $1_{i}, i<\aleph_{0}$, be the canonical basis of the free module $F=R^{\left(\aleph_{0}\right)}$ and let $G=\sum_{i<\aleph_{0}}\left(1_{i}-1_{i+1} \cdot a_{i}\right) R \subseteq F$. Put $M=F / G$. By Bass' lemma, $G$ is a free module, and $M$ is not projective. If $N$ is a finitely generated module, we have $N \simeq R^{(n)} / K$ for some $n<\aleph_{0}$ and $K \subseteq R^{(n)}$. Since the sequence $0 \rightarrow G \rightarrow F \rightarrow M \rightarrow 0$ is exact, we get $0=\operatorname{Ext}_{R}(G, K) \rightarrow \operatorname{Ext}_{R}^{2}(M, K) \rightarrow$ $\operatorname{Ext}_{R}^{2}(F, K)=0$, and $\operatorname{Ext}_{R}^{2}(M, K)=0$. Since the sequence $0 \rightarrow K \rightarrow R^{(n)} \rightarrow$ $N \rightarrow 0$ is exact and $R$ is right self-injective, we have $0=\operatorname{Ext}_{R}\left(M, R^{(n)}\right) \rightarrow$ $\operatorname{Ext}_{R}(M, N) \rightarrow \operatorname{Ext}_{R}^{2}(M, K)=0$, whence $\operatorname{Ext}_{R}(M, N)=0$.

For right hereditary rings, the question of existence of p-test modules can be decided on free modules:

Proposition 2.7. Let $R$ be a ring and $\kappa$ a cardinal. Then the following conditions are equivalent:

(i) There exists a p-test module $N$ such that $\operatorname{gen}(N) \leq \kappa$ and $\operatorname{proj} \cdot \operatorname{dim}(N) \leq 1$;

(ii) $R^{(\kappa)}$ is a p-test module.

Proof. The non-trivial part is (i) $\Longrightarrow$ (ii) : Let $M$ be a module such that $\operatorname{Ext}_{R}\left(M, R^{(\kappa)}\right)=0$. By the premise, there is an exact sequence $0 \rightarrow K \rightarrow R^{(\kappa)} \rightarrow$ 
$N \rightarrow 0$, where $K$ is projective. Then $0=\operatorname{Ext}_{R}\left(M, R^{(\kappa)}\right) \rightarrow \operatorname{Ext}_{R}(M, N) \rightarrow$ $\operatorname{Ext}_{R}^{2}(M, K)=0$, whence $\operatorname{Ext}_{R}(M, N)=0$, and $M$ is projective by (i).

Corollary 2.8. Let $R$ be a right hereditary ring. If there is a p-test module in $M o d-R$, then there is a cardinal $\kappa$ such that each free module of rank $\geq \kappa$ is p-test.

Proof. Take $\kappa=\min \{\operatorname{gen}(N) ; N$ is p-test $\}$ and apply 2.7 .

\section{Generalized Weak diamond And the existence of P-Test modules}

The main purpose of this section is to prove the consistency of the existence of p-test modules for certain classes of non-right perfect rings (Theorem 3.13(ii)). An essential tool for this is a combinatorial principle called generalized weak diamond (and denoted by $\Psi$ ). Since $\Psi$ is a consequence of the axiom of constructibility, all consequences of $\Psi$ are consistent with ZFC + GCH. Our proof is in three steps:

Step I: by purely algebraic means, the existence of modules testing projectivity of modules of "small" size is obtained;

Step II: using $\Psi$, the testing is extended to modules of regular cardinality;

Step III: Shelah's Compactness Theorem is applied to cover the singular cardinality case.

Note that the proof requires the generalized weak diamond only in Step II, the other steps being proved in ZFC.

In this way, the existence of p-test modules is achieved for all right hereditary non-right perfect rings. Further results are obtained in the particular cases when

(1) $R$ is a Dedekind domain with $\operatorname{card}(R) \leq \aleph_{1}$ such that $R$ is not a complete discrete valuation ring; and

(2) $R$ is a simple von Neumann regular ring with $\operatorname{card}(R) \leq \aleph_{1}$ such that $R$ has countable dimension over its center.

In the case (1), p-test modules include all non-zero free modules (Theorem 3.14). In the case (2), all non-zero countably generated modules are p-test. Hence, also all non-zero free modules, and semisimple modules, are p-test in the case (2) (Corollary $3.19)$.

Step I for the Dedekind domains is a well-known generalization of the classical result of Stein for $\mathbb{Z}$. The generalization is due to Nunke $([\mathrm{N}, \S 8])$ :

Proposition 3.1. Let $R$ be a Dedekind domain which is not a complete discrete valuation ring. Let $F$ be a non-zero free module, and $M$ be a module of countable rank. Then $M$ is projective iff $\operatorname{Ext}_{R}(M, F)=0$.

We turn to Step I for the von Neumann regular rings:

Lemma 3.2. Let $R$ be a von Neumann regular ring such that $\operatorname{dim}_{K}(R) \leq \aleph_{0}$, $K$ being the center of $R$. Then each left (right) ideal of $R$ is countably generated. Hence $R$ is (left and right) hereditary. In particular, any countable von Neumann regular ring is hereditary.

Proof. Let $I$ be a right ideal of $R$. Then $\operatorname{dim}_{K}(I) \leq \aleph_{0}$. Let $B=\left\{b_{n} ; n<\kappa\right\}$, $\kappa \leq \aleph_{0}$, be a left $K$-basis of $I$. Then $I=\sum_{n<\kappa} b_{n} R$, and $I$ is countably generated. Since $R$ is regular, $I$ is projective, and $R$ is right hereditary. The left-hand version follows similarly. 
Proposition 3.3. Let $R$ be a simple von Neumann regular ring. Denote by $K$ the center of $R$. Assume $\operatorname{dim}_{K}(R) \leq \aleph_{0}$. Let $N$ be a non-zero countably generated module. Let $M$ be any countably generated module. Then $M$ is projective iff $\operatorname{Ext}_{R}(M, N)=0$.

Proof. Let $\operatorname{Ext}_{R}(M, N)=0$. Let $M^{\prime}$ be any finitely generated submodule of $M$. We prove that $M^{\prime}$ is projective: We have $M^{\prime} \simeq R^{(m)} / I$ for some $0<m<\aleph_{0}$ and $I \in M o d-R$. Proving indirectly, assume $\operatorname{gen}(I) \geq \aleph_{0}$. Then the regularity of $R$ implies $I$ is a direct sum of cyclic modules, $I=\bigoplus \sum_{\alpha<\kappa} x_{\alpha} R$. Since $\operatorname{Ext}_{R}(M, N)=$ 0, 3.2 implies $\operatorname{Ext}_{R}\left(M^{\prime}, N\right)=0$. Then also $\operatorname{Ext}_{R}\left(R^{(m)} / I^{\prime}, N\right)=0$, where $I^{\prime}=$ $\bigoplus \sum_{\alpha \in C} x_{\alpha} R$, for a countably infinite subset $C \subseteq \kappa$. Since each $x_{\alpha} R$ is cyclic and projective, there exist idempotents $0 \neq e_{\alpha}, \alpha \in C$, such that $x_{\alpha} R \simeq e_{\alpha} R$ for all $\alpha \in C$. Since $R$ is a simple ring, we have $N e_{\alpha} \neq 0$ for all $\alpha \in C$. In particular, $\operatorname{dim}_{K}\left(\operatorname{Hom}_{R}\left(I^{\prime}, N\right)\right)=\operatorname{dim}_{K}\left(\prod_{\alpha \in C} N e_{\alpha}\right) \geq \operatorname{dim}_{K}\left(K^{\aleph_{0}}\right)>\aleph_{0}$, while $\operatorname{dim}_{K}\left(\operatorname{Hom}_{R}\left(R^{(m)}, N\right)\right)=\operatorname{dim}_{K}\left(N^{(m)}\right) \leq \aleph_{0}$. This contradicts the exactness of the sequence

$$
0 \rightarrow \operatorname{Hom}_{R}\left(R^{(m)} / I^{\prime}, N\right) \rightarrow \operatorname{Hom}_{R}\left(R^{(m)}, N\right) \rightarrow \operatorname{Hom}_{R}\left(I^{\prime}, N\right) \rightarrow 0 .
$$

Hence, $\operatorname{gen}(I)<\aleph_{0}, I$ is a summand of $R^{(m)}$ (as $R$ is regular), and $M^{\prime}$ is projective. Thus, $M$ is $\aleph_{0}$-projective, and the assertion is true provided $M$ is finitely generated. If $\operatorname{gen}(M)=\aleph_{0}$, we use the following

Lemma 3.4. Let $R$ be a right hereditary von Neumann regular ring. Let $M$ be an $\aleph_{0}$-projective module with gen $(M)=\aleph_{0}$. Then $M$ is projective.

Proof. We have $M=R^{\left(\aleph_{0}\right)} / I$ for some $I \subseteq R^{\left(\aleph_{0}\right)}$. Put $M_{n}=\left(R^{(n)}+I\right) / I$ and $I_{n}=R^{(n)} \cap I, n<\aleph_{0}$. Then $M$ is a union of the non-decreasing chain $\left(M_{n} ; n<\aleph_{0}\right)$. By the premise, $M_{n} \simeq R^{(n)} / I_{n}$ is projective, and $I_{n}$ is finitely generated. Therefore, we can define two sets, $\left(A_{n} ; 0<n<\aleph_{0}\right)$, and $\left(B_{n} ; 0<n<\aleph_{0}\right)$, of finitely generated submodules of $R^{\left(\aleph_{0}\right)}$ by

$$
B_{n} \oplus\left(I_{n}+R^{(n-1)}\right)=R^{(n)} \quad \text { and } \quad I_{n+1}=I_{n} \oplus A_{n}
$$

for each $0<n<\aleph_{0}$. Then $R^{\left(\aleph_{0}\right)}=I_{1} \oplus\left(\oplus \sum_{0<n<\aleph_{0}} A_{n}\right) \oplus\left(\bigoplus \sum_{0<n<\aleph_{0}} B_{n}\right)$. Now, $I=\bigcup_{0<n<\aleph_{0}} I_{n}, I=I_{1} \oplus\left(\bigoplus \sum_{0<n<\aleph_{0}} A_{n}\right)$, and $M \simeq \bigoplus \sum_{0<n<\aleph_{0}} B_{n}$ is projective.

For Step II, we start with combinatorial principles that follow from the axiom of constructibility:

Definition 3.5. Let $\kappa$ be a regular uncountable cardinal and $E$ be a stationary subset of $\kappa$. Denote by $\nabla_{\kappa}(E)$ the Jensen's diamond (for $\kappa$ and $E$ ), i.e. the assertion

" Let $A$ be any set of cardinality $\kappa$ and $\left(A_{\alpha} ; \alpha<\kappa\right)$ a $\kappa$-filtration of $A$. Then there is a system $\left\{S_{\alpha} ; \alpha<\kappa\right\}$ such that $S_{\alpha} \subseteq A_{\alpha}$ for all $\alpha<\kappa$, and the set $\left\{\alpha \in E ; X \cap A_{\alpha}=S_{\alpha}\right\}$ is stationary in $\kappa$, for every $X \subseteq A$."

Denote by $\Phi_{\kappa}(E)$ the weak diamond (for $\kappa$ and $E$ ), i.e. the assertion

"Let $A$ be any set of cardinality $\kappa$ and $\left(A_{\alpha} ; \alpha<\kappa\right)$ a $\kappa$-filtration of $A$. For each $\alpha \in E$, let $P_{\alpha}: \operatorname{Exp}\left(A_{\alpha}\right) \rightarrow\{0,1\}$ be given. Then there is $\phi: E \rightarrow\{0,1\}$ such that the set $\left\{\alpha \in E ; P_{\alpha}\left(X \cap A_{\alpha}\right)=\phi(\alpha)\right\}$ is stationary in $\kappa$, for every $X \subseteq A$."

Denote by $\Psi_{\kappa}(E)$ the assertion 
" Let $A$ be any set of cardinality $\kappa$ and $\left(A_{\alpha} ; \alpha<\kappa\right)$ a $\kappa$-filtration of $A$. For each $\alpha \in E$, let $2 \leq p_{\alpha}<\aleph_{0}$ and let $P_{\alpha}: \operatorname{Exp}\left(A_{\alpha}\right) \rightarrow p_{\alpha}$ be given. Then there is $\psi: E \rightarrow \aleph_{0}$ such that $\psi(\alpha) \in p_{\alpha}$ for all $\alpha \in E$, and the set $\left\{\alpha \in E ; P_{\alpha}\left(X \cap A_{\alpha}\right)=\right.$ $\psi(\alpha)\}$ is stationary in $\kappa$, for every $X \subseteq A$."

Finally, denote by $\Psi$ the generalized weak diamond, i.e. the assertion:

" $\Psi_{\kappa}(E)$ holds true for each regular uncountable cardinal $\kappa$ and each stationary subset $E \subseteq \kappa "$.

The generalized weak diamond principle is less well-known, so we notice its position between the better known ones:

Lemma 3.6. (i) Let $\kappa$ be a regular uncountable cardinal and $E$ be a stationary subset of $\kappa$. Then $\nabla_{\kappa}(E) \Longrightarrow \Psi_{\kappa}(E) \Longrightarrow \Phi_{\kappa}(E)$.

(ii) $\Psi$ is consistent with $Z F C+G C H$.

Proof. (i) This is clear, taking $\psi(\alpha)=P_{\alpha}\left(S_{\alpha}\right)$ for all $\alpha \in E$ for the first implication, and taking $p_{\alpha}=2$ for all $\alpha<\kappa$ for the second.

(ii) Assume the axiom of constructibility. Then, by a well-known result of Jensen, $\nabla_{\kappa}(E)$ holds for each regular uncountable cardinal $\kappa$ and each stationary subset $E$ of $\kappa$. Note that the Jensen's diamond for $\kappa=\lambda^{+}$and $E=\lambda^{+}$implies $2^{\lambda}=\lambda^{+}$. Hence, GCH holds, and (i) implies that the assertion holds true.

In general, by [S5, Ch.XIV], none of the implications from 3.6(i) can be reversed.

Similarly as the (weak) diamond (in [EM, XII, §1] et al.), the generalized weak diamond is very useful for investigations of vanishing of Ext:

Lemma 3.7. Let $\kappa$ be a regular uncountable cardinal and $E$ a stationary subset of $\kappa$. Assume $\Psi_{\kappa}(E)$. Let $R$ be a ring with $\operatorname{card}(R) \leq \kappa$. Let $N$ be a module such that $\operatorname{card}(I(N)) \leq \kappa$. Let $M$ be a $\kappa$-projective module such that $\operatorname{gen}(M)=\kappa$ and there is a $\kappa$-filtration $\left(C_{\alpha} ; \alpha<\kappa\right)$ of $M$ such that $E=\left\{\alpha<\kappa ; \operatorname{Ext}_{R}\left(C_{\alpha+1} / C_{\alpha}, N\right) \neq 0\right\}$. Then $\operatorname{Ext}_{R}(M, N) \neq 0$.

Proof. First, we take a $\kappa$-filtration $\left(D_{\alpha} ; \alpha<\kappa\right)$ of the set $\kappa$ and elements $m_{\alpha} \in M$, $\alpha<\kappa$, such that $C_{\alpha}=\sum_{\beta \in D_{\alpha}} m_{\beta} R$, for all $\alpha<\kappa$. Let $\left(B_{\alpha} ; \alpha<\kappa\right)$ be a $\kappa$ filtration of the $\mathbb{Z}$-module $I=I(N)$. Denote by $\nu$ the inclusion of $N$ into $I$, by $\pi$ the projection of $I$ onto $I / N$, and by $\nu_{\alpha}$ the inclusion of $C_{\alpha}$ into $C_{\alpha+1}$, for all $\alpha<\kappa$.

Take $\alpha \in E$. Let $X_{\alpha}=\operatorname{Hom}_{R}\left(C_{\alpha}, N\right)$ and $Y_{\alpha}=\operatorname{Im}\left(\operatorname{Hom}\left(\nu_{\alpha}, N\right)\right)$. By the premise, there is some $f_{\alpha} \in X_{\alpha} \backslash Y_{\alpha}$. Denote by $o_{\alpha}$ the order of $f_{\alpha}+Y_{\alpha}$ in the group $X_{\alpha} / Y_{\alpha}=\operatorname{Ext}_{R}\left(C_{\alpha+1} / C_{\alpha}, N\right)$.

We are going to use the principle $\Psi_{\kappa}(E)$ in the following setting: $A=\kappa \times I$ and $A_{\alpha}=D_{\alpha} \times B_{\alpha}, \alpha<\kappa$. Let $\alpha \in E$. If $o_{\alpha}=\aleph_{0}$, we put $p_{\alpha}=2$. If $o_{\alpha}<\aleph_{0}$, we define $p_{\alpha}=o_{\alpha}$. In order to define the colourings $P_{\alpha}, \alpha \in E$, we equip the set of all mappings from $D_{\alpha}$ to $B_{\alpha}$ with an equivalence relation $\sim_{\alpha}$ : we put $u \sim_{\alpha} v$ iff there are $n \in \mathbb{Z}$ and $y \in Y_{\alpha}$ such that $v=u+n f_{\alpha}\left\lceil D_{\alpha}+y\left\lceil D_{\alpha}\right.\right.$. Note that the number $n$ is unique (unique modulo $p_{\alpha}$ ) provided $o_{\alpha}=\aleph_{0}\left(o_{\alpha}<\aleph_{0}\right)$. Now, for each $\alpha \in E$, we take a colouring $P_{\alpha}: \operatorname{Exp}\left(A_{\alpha}\right) \rightarrow p_{\alpha}$ such that $P_{\alpha}(u)=P_{\alpha}(v)$ iff the number $n$ given by the pair $(u, v)$ is divisible by $p_{\alpha}$.

Let $\psi: E \rightarrow \aleph_{0}$ be the mapping corresponding to this setting by $\Psi_{\kappa}(E)$. In order to prove that $\operatorname{Ext}_{R}(M, N) \neq 0$, we shall construct

$$
g \in \operatorname{Hom}_{R}(M, I / N) \backslash \operatorname{Im}\left(\operatorname{Hom}_{R}(M, \pi)\right) .
$$


By induction on $\alpha<\kappa$, we define $g_{\alpha} \in \operatorname{Hom}_{R}\left(C_{\alpha}, I / N\right)$ so that $g_{\alpha+1}\left\lceil C_{\alpha}=g_{\alpha}\right.$ for each $\alpha<\kappa$, and $g_{\alpha}=\bigcup_{\beta<\alpha} g_{\beta}$ for all limit $\alpha<\kappa$.

Put $g_{0}=0$. Assume $g_{\alpha}$ is defined for an ordinal $\alpha<\kappa$. We distinguish the following two cases:

(I) $\alpha \in E$ and there exists $f \in \operatorname{Hom}_{R}\left(C_{\alpha+1}, I\right)$ such that $\operatorname{Im}\left(f \nu_{\alpha} \uparrow D_{\alpha}\right) \subseteq B_{\alpha}$, $P_{\alpha}\left(f \nu_{\alpha} \uparrow D_{\alpha}\right)=\psi(\alpha)$, and $g_{\alpha}=\pi f \nu_{\alpha}$.

(II) $=\operatorname{not}(\mathrm{I})$.

In the case (I), take an $f$ satisfying the conditions of (I). The injectivity of $I$ yields the existence of $h_{\alpha} \in \operatorname{Hom}_{R}\left(C_{\alpha+1}, I\right)$ such that $h_{\alpha} \nu_{\alpha}=f \nu_{\alpha}-f_{\alpha}$. Put $g_{\alpha+1}=\pi h_{\alpha}$. Then $g_{\alpha+1} \nu_{\alpha}=\pi f \nu_{\alpha}-\pi f_{\alpha}=g_{\alpha}$.

In the case (II), the projectivity of $C_{\alpha}$ yields the existence of $h_{\alpha} \in H_{0} m_{R}\left(C_{\alpha}, I\right)$ such that $g_{\alpha}=\pi h_{\alpha}$. The injectivity of $I$ gives some $h_{\alpha+1} \in \operatorname{Hom}_{R}\left(C_{\alpha+1}, I\right)$ such that $h_{\alpha}=h_{\alpha+1} \nu_{\alpha}$. Put $g_{\alpha+1}=\pi h_{\alpha+1}$. Then $g_{\alpha+1}\left\lceil C_{\alpha}=g_{\alpha}\right.$.

Finally, put $g=\bigcup_{\alpha<\kappa} g_{\alpha}$. Then $g \in \operatorname{Hom}_{R}(M, I / N)$. Proving indirectly, suppose there is $h^{\prime} \in \operatorname{Hom}_{R}(M, I)$ such that $g=\pi h^{\prime}$. Note that the set $\{\alpha<\kappa$; $\left.\operatorname{Im}\left(h^{\prime} \uparrow D_{\alpha}\right) \subseteq B_{\alpha}\right\}$ is closed and cofinal in $\kappa$. Put $X=\bigcup_{\alpha<\kappa}\left(h^{\prime} \uparrow D_{\alpha}\right)$. By the premise, there is an $\alpha \in E$ such that $g\left\lceil C_{\alpha}=\pi h \nu_{\alpha}, P_{\alpha}\left(h \nu_{\alpha}\left\lceil D_{\alpha}\right)=P_{\alpha}\left(X \cap A_{\alpha}\right)=\right.\right.$ $\psi(\alpha)$, and $\operatorname{Im}\left(h \nu_{\alpha}\left\lceil D_{\alpha}\right) \subseteq B_{\alpha}\right.$, where $h=h^{\prime}\left\lceil C_{\alpha+1}\right.$. Hence, the case (I) occurs, and $\pi\left(h_{\alpha}-h\right)=0$. Then $y_{\alpha}=\left(h_{\alpha}-h\right) \nu_{\alpha} \in Y_{\alpha}$. Moreover, $f \nu_{\alpha}=h \nu_{\alpha}+f_{\alpha}+y_{\alpha}$, whence $\psi(\alpha)=P_{\alpha}\left(f \nu_{\alpha}\left\lceil D_{\alpha}\right)=P_{\alpha}\left(h \nu_{\alpha}\left\lceil D_{\alpha}+f_{\alpha}\left\lceil D_{\alpha}+y_{\alpha}\left\lceil D_{\alpha}\right) \neq P_{\alpha}\left(h \nu_{\alpha}\right\rceil\right.\right.\right.\right.$ $\left.D_{\alpha}\right)$, a contradiction. Thus $g \notin \operatorname{Im}\left(\operatorname{Hom}_{R}(M, \pi)\right)$.

Lemma 3.8. Let $\kappa$ be a regular uncountable cardinal. Assume $\Psi_{\kappa}(E)$ holds for all stationary subsets of $\kappa$. Let $R$ be a ring with $\operatorname{card}(R) \leq \kappa$. Let $N$ be a module such that $\operatorname{card}(I(N)) \leq \kappa$. Let $M$ be a $\kappa$-projective module such that $\operatorname{gen}(M)=\kappa$. Then the following conditions are equivalent:

(i) $\operatorname{Ext}_{R}(M, N)=0$;

(ii) There is a $\kappa$-filtration $\left(C_{\alpha} ; \alpha<\kappa\right)$ of $M$ such that $\operatorname{Ext}_{R}\left(C_{\alpha+1} / C_{\alpha}, N\right)=0$ for all $\alpha<\kappa$.

Moreover, the implication (ii) $\Longrightarrow$ (i) holds in ZFC.

Proof. (i) $\Longrightarrow$ (ii): Since $\operatorname{gen}(M)=\kappa$, there is a $\kappa$-filtration, $\left(D_{\alpha} ; \alpha<\kappa\right)$, of the module $M$. By induction, we define a mapping $\eta: \kappa \rightarrow \kappa$ as follows. First, $\eta(0)=0$. If $\eta(\alpha)$ is defined, then either $\operatorname{Ext}_{R}\left(D_{\beta} / D_{\eta(\alpha)}, N\right)=0$ for all $\beta \geq \eta(\alpha)$ and we put $\eta(\alpha+1)=\eta(\alpha)+1$, or there is a smallest $\eta(\alpha)<\beta<\kappa$ such that $\operatorname{Ext}_{R}\left(D_{\beta} / D_{\eta(\alpha)}, N\right) \neq 0$ and we put $\eta(\alpha+1)=\beta$. For $\alpha$ limit, we put $\eta(\alpha)=\sup _{\beta<\alpha} \eta(\beta)$. Then $\left(D_{\eta(\alpha)} ; \alpha<\kappa\right)$ is a $\kappa$-filtration of the module $M$. Let $E=\left\{\alpha<\kappa ; \operatorname{Ext}_{R}\left(D_{\eta(\alpha+1)} / D_{\eta(\alpha)}, N\right) \neq 0\right\}$. By 3.7, $E$ is not stationary in $\kappa$. Let $C$ be a closed and cofinal subset of $\kappa$ with $E \cap C=\emptyset$ and $0 \in C$. Let $\theta: \kappa \rightarrow C$ be a strictly increasing continuous mapping of $\kappa$ onto $C$. For each $\alpha<\kappa$, put $C_{\alpha}=D_{\eta \theta(\alpha)}$. Then $\left(C_{\alpha} ; \alpha<\kappa\right)$ is a $\kappa$-filtration of $M$ satisfying (ii).

(ii) $\Longrightarrow$ (i): We prove in ZFC. Denote by $\pi$ the projection of $I=I(N)$ onto $I / N$. For $\alpha<\kappa$, let $\nu_{\alpha}$ be the inclusion of $C_{\alpha}$ into $C_{\alpha+1}$. By induction on $\alpha<\kappa$ we define mappings $\varphi_{\alpha}: \operatorname{Hom}_{R}\left(C_{\alpha}, I / N\right) \rightarrow \operatorname{Hom}_{R}\left(C_{\alpha}, I\right)$ such that for each $f \in \mathrm{Hom}_{R}\left(C_{\alpha}, I / N\right), \pi \varphi_{\alpha}(f)=f$, and $\varphi_{\alpha}\left(f \nu_{\alpha}\right)=\varphi_{\beta}(f) \nu_{\alpha}$ provided $\beta=\alpha+1$. For $\alpha=0$ put $\varphi_{\alpha}=0$. Let $0<\alpha<\kappa$ and $f \in \operatorname{Hom}_{R}\left(C_{\alpha+1}, I / N\right)$. Since $C_{\alpha+1}$ is projective, there is $g \in \operatorname{Hom}_{R}\left(C_{\alpha+1}, I\right)$ with $f=\pi g$. As $g \nu_{\alpha}-\varphi_{\alpha}\left(f \nu_{\alpha}\right) \in$ $\operatorname{Hom}_{R}\left(C_{\alpha}, N\right)$ and $\operatorname{Ext}_{R}\left(C_{\alpha+1} / C_{\alpha}, N\right)=0$, there is $h \in \operatorname{Hom}_{R}\left(C_{\alpha+1}, N\right)$ such that $h \nu_{\alpha}=g \nu_{\alpha}-\varphi_{\alpha}\left(f \nu_{\alpha}\right)$. We put $\varphi_{\alpha+1}(f)=g-h$. If $\alpha$ is limit, define $\varphi_{\alpha}=$ 
$\bigcup_{\beta<\alpha} \varphi_{\beta}$. Then $f=\pi \varphi_{\alpha}(f)$, for all $f \in \operatorname{Hom}_{R}\left(C_{\alpha}, I / N\right)$. Finally, put $\varphi=$ $\bigcup_{\alpha<\kappa} \varphi_{\alpha}$. Then $f=\pi \varphi(f)$, for all $f \in \operatorname{Hom}_{R}\left(C_{\alpha}, I / N\right)$, and $\operatorname{Ext}_{R}(M, N)=0$.

Though our main applications will be to hereditary rings, the rings $R$ in 3.7 and 3.8 need not be right hereditary in general (consider $R=R^{\prime} \boxplus R^{\prime \prime}$, where $R^{\prime}$ is right hereditary, but $R^{\prime \prime}$ is not, and let $M, N \in \operatorname{Mod}-R^{\prime} \subset \operatorname{Mod}-R$ satisfy the conditions of 3.7 and 3.8$)$.

Definition 3.9. Let $M$ be a module and $\lambda \geq \aleph_{0}$. Assume that for some submodules of $M$, sets called "bases" are given. If $N$ is a submodule of $M$ such that $N$ has at least one "basis", we say that $N$ is "free". We introduce the following axioms:

(Ax I) If $N$ is a "free" submodule of $M$ and $\mathcal{F}$ is a "basis" of $N$, then $\mathcal{F}$ is a set of submodules of $N, \mathcal{F}$ is closed under unions of chains, and for each subset $A \subseteq N$ there is some $F \in \mathcal{F}$ such that $A \subseteq F$ and $\operatorname{card}(F) \leq \operatorname{card}(A)+\lambda$.

(Ax II) If $N$ is a "free" submodule of $M, \mathcal{F}$ is a "basis" of $N$ and $C \in \mathcal{F}$, then $\mathcal{F}\lceil C=\{D \in \mathcal{F} ; D \subseteq C\}$ is a "basis" of $C$.

(Ax III) If $N$ is a "free" submodule of $M, C$ is an element of a "basis" of $N$, and $C$ has a "basis" $\mathcal{G}$, then $N$ has a "basis" $\mathcal{F}$ such that $\mathcal{G}=\mathcal{F}\lceil C$.

(Ax IV) Suppose $\left(N_{\alpha} ; \alpha<\lambda\right)$ is a smooth chain of "free" submodules of $M$; for each $\alpha<\lambda$ a "basis" $\mathcal{F}_{\alpha}$ of $N_{\alpha}$ is given so that $\alpha<\beta<\lambda$ implies $\mathcal{F}_{\alpha}=\mathcal{F}_{\beta}\left\lceil N_{\alpha}\right.$. Then $\bigcup_{\alpha<\lambda} N_{\alpha}$ has a "basis" consisting of all sets of the form $\bigcup_{\alpha<\lambda} C_{\alpha}$, where $\left(C_{\alpha} ; \alpha<\lambda\right)$ is a chain of submodules of $M$, and $C_{\alpha} \in \mathcal{F}_{\alpha}$ for all $\alpha<\lambda$.

Now, we formulate the version of Shelah's Compactness Theorem that we shall need for Step III. Its proof, using game theoretic arguments, appears e.g. in [Ho, $\S 4]$ or [EM, Ch.IV]:

Theorem 3.10. Let $R$ be a ring and $M$ be a module such that $\operatorname{card}(M)=\kappa$ is a singular cardinal. Let $\lambda$ be an infinite cardinal such that $\operatorname{card}(R) \leq \lambda<\kappa$. Assume the axioms (Ax I)-(Ax IV) from 3.8 hold, and every submodule of $M$ of cardinality $<\kappa$ is "free". Then $M$ is "free".

Corollary 3.11. Let $R$ be a ring and $M$ be a module such that $\operatorname{card}(M)=\kappa$ is a singular cardinal. Assume that $\operatorname{card}(R)<\kappa$ and $M$ is $\kappa$-projective. Then $M$ is projective.

Proof. We shall say that a submodule $N$ of $M$ is "free" provided there are a cardinal $\mu$ and countably generated projective modules $P_{\alpha} \subseteq N, \alpha<\mu$, such that $N=$ $\bigoplus \sum_{\alpha<\mu} P_{\alpha}$. Then the set $\mathcal{F}=\left\{C ; \exists A \subseteq \mu: \bar{C}=\oplus \sum_{\alpha \in A} P_{\alpha}\right\}$ is called a "basis" of the module $N$. Put $\lambda=\operatorname{card}(R) \times \aleph_{0}$. Then each countably generated projective module has cardinality $\leq \lambda$. It is easy to see that the notions in quotes satisfy axioms (Ax I)-(Ax IV) of 3.9. On the other hand, Kaplansky's structure theorem for projective modules implies that a module is "free" iff it is projective. Hence, the premise and 3.10 imply that $M$ is projective.

Combining Steps II and III, we obtain

Theorem 3.12. Assume $\Psi$. Let $R$ be a right hereditary ring with $\operatorname{card}(R) \leq \aleph_{1}$. Let $N$ be a module such that card $(I(N)) \leq \aleph_{1}$. Assume that $\operatorname{Ext}_{R}(M, N)=0$ implies $M$ is projective, for every countably generated module $M$. Then $N$ is a p-test module.

Proof. By induction on $\operatorname{gen}(M)=\kappa$, we prove that $M$ is projective whenever $M$ is a module such that $\operatorname{Ext}_{R}(M, N)=0$. If $\kappa \leq \aleph_{0}$, the assertion holds by the premise. 
Let $\kappa$ be a regular uncountable cardinal. Since $R$ is right hereditary, 3.8 implies $M$ has a $\kappa$-filtration $\left(C_{\alpha} ; \alpha<\kappa\right)$ such that $\operatorname{Ext}_{R}\left(C_{\alpha+1} / C_{\alpha}, N\right)=0$ for all $\alpha<\kappa$. By the induction premise, all the modules $C_{\alpha+1} / C_{\alpha}, \alpha<\kappa$, are projective, whence $M=\bigcup_{\alpha<\kappa} C_{\alpha}$ is projective.

Let $\kappa$ be singular. Since $R$ is right hereditary, $M$ is $\kappa$-projective, by the induction premise. Now, 3.11 terminates the proof.

For right hereditary rings, 2.8 shows that the existence of p-test modules implies the existence of free $\mathrm{p}$-test modules. This occurs under $\Psi$ :

Theorem 3.13. Assume $\Psi$. Let $R$ be a right hereditary non-right perfect ring.

(i) Let $\lambda \geq \aleph_{0}$ be a cardinal such that card $\left(I\left(R^{(\lambda)}\right)\right) \leq \lambda$. Then each free module of rank $\geq \lambda$ is p-test.

(ii) Each free module of rank $\geq 2^{\operatorname{card}(R)}$ is p-test.

Proof. (i) Assume $M$ is a $\leq \lambda$ generated module such that $\operatorname{Ext}_{R}\left(M, R^{(\lambda)}\right)=0$. Let $K$ be a submodule of $R^{(\lambda)}$ such that $M \simeq R^{(\lambda)} / K$. By the premise, $\operatorname{gen}(K) \leq \lambda$. Since $R$ is right hereditary, we infer that $\operatorname{Ext}_{R}(M, K)=0$, and $M$ is projective by 1.1(i). Now, starting from $\lambda$, and using 3.8 and 3.11 for induction in regular and singular cardinals, respectively, we obtain the claim.

(ii) Since $R$ is not right perfect, we have $\kappa=\operatorname{card}(R) \geq \aleph_{0}$. We prove that $\operatorname{card}\left(I\left(R^{\left(2^{\kappa}\right)}\right)\right) \leq 2^{\kappa}$. Put $M=R^{\left(2^{\kappa}\right)}$. Then there is a chain of modules

$$
M \simeq \operatorname{Hom}_{R}(R, M) \subseteq \operatorname{Hom}_{\mathbb{Z}}(R, M) \subseteq \operatorname{Hom}_{\mathbb{Z}}(R, D)=I,
$$

where $D$ is the divisible hull of the (right) $\mathbb{Z}$-module $M$. Since $R$ is a flat left $R$-module, $I$ is injective. Since $\operatorname{card}(D)=\operatorname{card}(M)=2^{\kappa}$, we infer that $\operatorname{card}(I) \leq$ $\left(2^{\kappa}\right)^{\kappa}=2^{\kappa}$. This proves $\operatorname{card}\left(I\left(R^{\left(2^{\kappa}\right)}\right)\right) \leq 2^{\kappa}$, and (i) applies.

Stronger results hold true for the particular cases of Dedekind domains and of von Neumann regular rings:

Theorem 3.14. Assume $\Psi$. Let $R$ be a Dedekind domain such that $R$ is not a complete discrete valuation ring, and $\operatorname{card}(R) \leq \aleph_{1}$. Then any non-zero free module is p-test.

Proof. It suffices to prove that $R$ is p-test. Denote by $K$ the quotient field of $R$. Then $K$ is an injective module, $R \subseteq K$, and $\operatorname{card}(K)=\operatorname{card}(R) \leq \aleph_{1}$. Hence, 3.1 and 3.12 show that $R$ is a p-test module.

Theorem 3.15. Assume $\Psi$. Let $R$ be a right hereditary von Neumann regular ring such that $\operatorname{card}\left(I\left(R^{\left(\aleph_{0}\right)}\right)\right) \leq \aleph_{1}$. Then each free module of rank $\geq \aleph_{0}$ is p-test.

Proof. By 3.4 and 3.12, we have to prove that $\operatorname{Ext}_{R}\left(M, R^{\left(\aleph_{0}\right)}\right)=0$ implies $M$ is projective for each finitely generated module $M$. Let $M \simeq R^{(n)} / K$. Since $R$ is regular, there are a cardinal $\kappa$ and elements $0 \neq x_{\alpha} \in K, \alpha<\kappa$, such that $K=\bigoplus \sum_{\alpha<\kappa} x_{\alpha} R$.

Proving indirectly, assume $\kappa \geq \aleph_{0}$. Take a system of pairwise disjoint sets $A_{k}, k<\aleph_{0}$, such that $\operatorname{card}\left(A_{k}\right)=n$ for each $k<\aleph_{0}$, and $\aleph_{0}=\bigcup_{k<\aleph_{0}} A_{k}$. For each $k<\aleph_{0}$, we identify $R^{(n)}$ with $R^{\left(A_{k}\right)}$ via an $R$-isomorphism $\nu_{k}$. Define $f \in$ $\operatorname{Hom}_{R}\left(K, R^{\left(\aleph_{0}\right)}\right)$ by $f\left(x_{\alpha}\right)=\nu_{\alpha}\left(x_{\alpha}\right)$ provided $\alpha<\aleph_{0}$, and by $f\left(x_{\alpha}\right)=0$ otherwise. Let $g \in \operatorname{Hom}_{R}\left(R, R^{\left(\aleph_{0}\right)}\right)$. Then $\operatorname{Im}(g) \subseteq R^{(m)}$ for some $m<\aleph_{0}$, and $g \uparrow K \neq f$. Then $\operatorname{Ext}_{R}\left(M, R^{\left(\aleph_{0}\right)}\right) \neq 0$, a contradiction.

Hence, $\kappa$ is finite, and $M$ is projective (as $R$ is regular). 
Lemma 3.16. Let $R$ be a von Neumann regular ring. Let $N$ be a module and $\lambda$ be a cardinal of cofinality $\omega$. Denote by $\pi_{\nu}$ the $\nu$-th canonical projection of $M^{\lambda}$ to $M, \nu<\lambda$. Let $\left\{\lambda_{k} ; k<\aleph_{0}\right\}$ be a cofinal subset of $\lambda$. For each $k<\aleph_{0}$ put $M_{k}=\left\{m \in M^{\lambda} ; \pi_{\nu}(m)=0 \forall \nu \geq \lambda_{k}\right\}$, and $M_{\lambda}=\bigcup_{k<\aleph_{0}} M_{k}$. Let $J$ be a countably generated right ideal of $R$. Then $\operatorname{Ext}_{R}\left(R / J, M^{\lambda} / M_{\lambda}\right)=0$.

Proof. If $J$ is finitely generated, then $R / J$ is projective (as $R$ is regular), and the assertion is clear. If $\operatorname{gen}(J)=\aleph_{0}$, the regularity of $R$ implies there is a set, $\left\{e_{n} ; n<\aleph_{0}\right\}$, of orthogonal idempotents of $R$ such that $J=\bigoplus \sum_{n<\aleph_{0}} e_{n} R$. We have to extend each $\varphi \in \operatorname{Hom}_{R}\left(J, M^{\lambda} / M_{\lambda}\right)$ to some $\phi \in \operatorname{Hom}_{R}\left(R, M^{\lambda} / M_{\lambda}\right)$. We have $\varphi\left(e_{n}\right)=\left(x_{\alpha}^{n} \cdot e_{n} ; \alpha<\lambda\right)+N_{\lambda}$ for some $x_{\alpha}^{n} \in N, n<\aleph_{0}, \alpha<\lambda$. For $\alpha<\lambda_{0}$, put $y_{\alpha}=0$. If $\lambda_{k} \leq \alpha<\lambda_{k+1}$, put $y_{\alpha}=\sum_{n \leq k} x_{\alpha}^{n} \cdot e_{n}$. Define $\phi \in \operatorname{Hom}_{R}\left(R, M^{\lambda} / M_{\lambda}\right)$ by $\phi(1)=\left(y_{\alpha} ; \alpha<\lambda\right)$. Since the idempotents $e_{n}, n<\aleph_{0}$ are orthogonal, we have $\phi\lceil J=\varphi$.

Proposition 3.17. Assume $\Psi$ and $C H$. Let $R$ be a von Neumann regular ring such that each right ideal is countably generated and $\operatorname{card}(R) \leq \aleph_{1}$. Let $N$ be a module such that $\operatorname{gen}(N) \leq \aleph_{1}$. Assume that $\operatorname{Ext}_{R}(M, N)=0$ implies $M$ is projective, for every finitely generated module $M$. Then $N$ is a p-test module.

Proof. First, we use 3.16 for $\lambda=\aleph_{0}$ and $\lambda_{n}=n, n<\aleph_{0}$. Then $N_{\lambda}=N^{\left(\aleph_{0}\right)}$. Put $I=N^{\aleph_{0}} / N^{\left(\aleph_{0}\right)}$. By Baer's Criterion and 3.16, $I$ is injective. Denote by $\nu$ the mapping assigning each $x \in N$ the coset of the constant sequence $\left(x ; k<\aleph_{0}\right)$. Then $\nu$ is an embedding of $N$ into $I$. Note that $\operatorname{card}(I) \leq \aleph_{1}^{\aleph_{0}}=\left(2^{\aleph_{0}}\right)^{\aleph_{0}}=\aleph_{1}$. So the injective hull $I(N)$ of $N$ has cardinality at most $\aleph_{1}$. Finally, the regularity of $R$ and 3.4 show that we can apply 3.12 .

Corollary 3.18. Assume $\Psi$ and $C H$. Let $R$ be a von Neumann regular ring such that each right ideal is countably generated and $\operatorname{card}(R) \leq \aleph_{1}$. Then each free module of rank $\geq \aleph_{0}$ is $p$-test.

Proof. Since $\operatorname{card}(R) \leq \aleph_{1}$, also $\operatorname{card}\left(I\left(R^{\left(\aleph_{0}\right)}\right)\right) \leq \aleph_{1}$, and we use 3.15 .

The main application of 3.17 is to the case when $R$ is simple and of countable dimension over its center:

Corollary 3.19. Assume $\Psi$ and $C H$. Let $R$ be a simple von Neumann regular ring such that $\operatorname{card}(R) \leq \aleph_{1}$ and $\operatorname{dim}_{K}(R) \leq \aleph_{0}, K$ being the center of $R$. Let $N$ be a non-zero module such that $N$ is either (i) countably generated, or (ii) projective, or (iii) semisimple. Then $N$ is a p-test module.

Proof. Part (i) follows from 3.2, 3.3 and 3.17. Parts (ii) and (iii) follow from the fact that the respective modules possess non-zero cyclic summands.

There is no analogue (in ZFC) to 3.13-19 for arbitrary non-right perfect rings:

Example 3.20. Let $\kappa$ be an uncountable cardinal, $K$ be a skew-field and $M$ be a right linear $K$-space of dimension $\kappa$ over $K$. Let $R$ be the ring of all linear transformations of $M$. Then no module $N$ with $\operatorname{proj} \operatorname{dim}(N) \leq 1$ is p-test. In particular, no free module is p-test.

Proof. Let $\left\{b_{\alpha} ; \alpha<\kappa\right\}$ be a basis of $M$. Define a system of idempotents $\left\{e_{\alpha} ; \alpha<\right.$ $\left.\aleph_{1}\right\}$ of $R$ as follows: $e_{\alpha}\left(b_{\beta}\right)=b_{\beta}$ provided $\beta \leq \alpha$, and $e_{\alpha}\left(b_{\beta}\right)=0$ otherwise. Define a chain of right ideals of $R$ by $I_{0}=0, I_{\alpha+1}=e_{\alpha} R, \alpha<\aleph_{1}$, and by $I_{\alpha}=\bigcup_{\beta<\alpha} I_{\beta}$ 
provided $\alpha$ is a limit ordinal $<\aleph_{1}$. Then $\left(I_{\alpha} ; \alpha<\aleph_{1}\right)$ is an $\aleph_{1}$-filtration of the right ideal $I=\bigcup_{\alpha<\aleph_{1}} I_{\alpha}$. Since $R$ is von Neumann regular, $I_{\alpha+1}$ is a summand in $I_{\beta}$ for all $\alpha<\beta<\aleph_{1}$. Hence, the set

$$
A=\left\{\alpha<\aleph_{1} ;\left\{\alpha<\beta<\aleph_{1} ; I_{\beta} / I_{\alpha} \text { is not projective }\right\} \text { is stationary in } \aleph_{1}\right\}
$$

contains all limit ordinals $<\aleph_{1}$, and $A$ is a stationary subset of $\aleph_{1}$. Applying [EM, IV, Proposition 1.7], we see that $I$ is a non-projective right ideal of $R$.

We prove that $\operatorname{Ext}_{R}\left(R / I, R^{(\lambda)}\right)=0$ for each cardinal $\lambda$. Let $\phi \in \operatorname{Hom}_{R}\left(I, R^{(\lambda)}\right)$. For each $\alpha<\aleph_{1}$, denote by $F_{\alpha}$ the smallest finite subset of $\lambda$ such that $\phi\left(e_{\alpha}\right) \in$ $R^{\left(F_{\alpha}\right)}$. Then $\left(F_{\alpha} ; \alpha<\aleph_{1}\right)$ is a non-decreasing chain of finite subsets of $\lambda$. Since $c f\left(\aleph_{1}\right)>\omega$, we infer that $F=\bigcup_{\alpha<\aleph_{1}} F_{\alpha}$ is a finite set, and $\operatorname{Im}(\phi) \subseteq R^{(F)}$. Since $R$ is right self-injective, there is some $\varphi \in \operatorname{Hom}_{R}\left(R, R^{(F)}\right)$ such that $\varphi \uparrow I=\phi$. Then $\operatorname{Ext}_{R}\left(R / I, R^{(\lambda)}\right)=0$, and $R^{(\lambda)}$ is not p-test.

Finally, by 2.7 , no module $N$ with $\operatorname{proj} \operatorname{dim}(N) \leq 1$ is p-test.

\section{Rings POSSESSing MANY TEST MODUleS}

By $\S 1$, we know that there is a proper class of i-test modules over any ring $R$. This suggests the question of how close can $\mathcal{I} \mathcal{T}$ be to Mod- $R$. Obviously, $\mathcal{I} \mathcal{T}=$ Mod- $R$ if and only if $R$ is semisimple. If $R$ is not semisimple, then no projective module is i-test. Thus, investigating the possible size of $\mathcal{I} \mathcal{T}$, we start with the question whether $\mathcal{I} \mathcal{T}$ can contain all "small" non-projective modules.

Since similar observations apply to the dual case of the class $\mathcal{P} \mathcal{T}$, we arrive at the following

Definition 4.1. Let $R$ be a non-semisimple ring and $\kappa$ be a cardinal. Then $\mathcal{I} \mathcal{T}$ $(\mathcal{P} \mathcal{T})$ is said to be $\kappa$-saturated provided it contains all non-projective (non-injective) modules $M$ such that $\operatorname{gen}(M) \leq \kappa$. Moreover, $\mathcal{I} \mathcal{T}(\mathcal{P} \mathcal{T})$ is fully saturated, provided it is $\kappa$-saturated for each $\kappa$.

A ring $R$ is said to be $\kappa$-saturated (fully saturated) provided the class $\mathcal{I} \mathcal{T}$ is such. Trivially, $\mathcal{I} \mathcal{T}$ and $\mathcal{P} \mathcal{T}$ are always 0 -saturated, whence any $\operatorname{ring} R$ is 0 -saturated. Also, $\kappa$-saturated implies $\kappa^{\prime}$-saturated for all $\kappa^{\prime} \leq \kappa$. Moreover, $\mathcal{I} \mathcal{T}$ is fully saturated iff $\mathcal{I} \mathcal{T}=\operatorname{Mod}-R \backslash \mathcal{P}$. Similarly, $\mathcal{P} \mathcal{T}$ is fully saturated iff $\mathcal{P} \mathcal{T}=\operatorname{Mod}-R \backslash \mathcal{I}$. Since each of these conditions is equivalent to the assertion " $\operatorname{Ext}_{R}(M, N) \neq 0$ whenever $M$ is non-projective and $N$ is non-injective", the two conditions are equivalent (to $R$ being fully saturated).

Note that fully saturated rings have been studied (under the term "right Extrings") in [T1]. Most of Sections 4-6 deals with the structure of $\kappa$-saturated rings for $\kappa=1$ and $\kappa=\aleph_{0}$, substantially refining and generalizing the results of [T1]. Moreover, our results show that even the condition of $R$ being 1-saturated imposes very strong restrictions on the structure of $R$. That is, "almost" no $R$ satisfies this condition. Of course, $R$ is 1-saturated iff $\mathcal{I} \mathcal{T}$ contains all cyclic non-projective modules iff $\operatorname{Ext}_{R}(R / J, N)=0$ implies $N$ is injective for all right ideals $J$ such that $J$ is not a summand of $R$. So by Baer's Criterion, our results show that for "almost" all rings $R$ there exist right ideals $I$ and $J$ and a module $N$ such that $J$ is not a summand of $R, \operatorname{Ext}_{R}(R / I, N) \neq 0$, but $\operatorname{Ext}_{R}(R / J, N)=0$.

We start with showing that if $\mathcal{I} \mathcal{T}$ contains all cyclic non-projective modules, then $R$ has no uncountably generated right ideals: 
Theorem 4.2. Let $R$ be a 1-saturated ring. Then each right ideal is countably generated. Moreover, either

(i) $R$ is right noetherian (i.e. each right ideal is finitely generated), or

(ii) $R$ is von Neumann regular.

Proof. (i) Assume $R$ is not von Neumann regular. Then there is a principal right ideal $r R$ which is not a summand in $R$. Let $\left(I_{\alpha} ; \alpha<\kappa\right)$ be any system of injective modules. Put $M=\bigoplus \sum_{\alpha<\kappa} I_{\alpha}$. We show that $M$ is injective. First, we prove that $\operatorname{Ext}_{R}(R / r R, M)=0$. Let $\phi \in H_{R}(r R, M)$. Then there is a finite set $F \subseteq \kappa$ such that $\operatorname{Im}(\phi) \subseteq \bigoplus \sum_{\alpha \in F} I_{\alpha}=M_{F}$. Since $M_{F}$ is injective, there is some $\varphi \in \operatorname{Hom}_{R}\left(R, M_{F}\right)$ such that $\varphi\left\lceil r R=\phi\right.$. Thus, $\operatorname{Ext}_{R}(R / r R, M)=0$. Since $R / r R$ is an i-test module, $M$ is injective. Hence, a direct sum of any system of injective right modules is injective, and (i) holds.

(ii) Assume $R$ is von Neumann regular, but not right noetherian. Then $R$ is not semisimple and there is an infinite set, $\left\{e_{n} ; n<\aleph_{0}\right\}$, of orthogonal idempotents in $R$. Put $J=\bigoplus \sum_{n<\aleph_{0}} e_{n} R$ and $M=R / J$. Then $J$ is projective and $M$ is a cyclic non-projective module.

Let $I$ be an injective module and $K$ be a submodule of $I$. Put $N=I / K$. Since the sequence $0 \rightarrow J \rightarrow R \rightarrow M \rightarrow 0$ is exact, we have

$$
0=\operatorname{Ext}_{R}(J, K) \rightarrow \operatorname{Ext}_{R}^{2}(M, K) \rightarrow \operatorname{Ext}_{R}^{2}(R, K)=0,
$$

and $\operatorname{Ext}_{R}^{2}(M, K)=0$. Since the sequence $0 \rightarrow K \rightarrow I \rightarrow N \rightarrow 0$ is exact, we have

$$
0=\operatorname{Ext}_{R}(M, I) \rightarrow \operatorname{Ext}_{R}(M, N) \rightarrow \operatorname{Ext}_{R}^{2}(M, K)=0
$$

and $\operatorname{Ext}_{R}(M, N)=0$. As $M$ is i-test, we infer that $N=I / K$ is injective. This proves that any factor module of an injective module is injective, and $R$ is right hereditary.

Now, we shall show that each right ideal is countably generated. On the contrary, assume there is a right ideal $I$ such that $\operatorname{gen}(I)>\aleph_{0}$. Since $R$ is right hereditary, $I=\bigoplus \sum_{\alpha<\kappa} x_{\alpha} R$ for an uncountable cardinal $\kappa$ and some $0 \neq x_{\alpha} \in R, \alpha<\kappa$ Put $J=\bigoplus \sum_{\alpha<\aleph_{0}} x_{\alpha} R$.

Let $M$ be a non-injective module. Let $H=\operatorname{Hom}_{R}(I, M)$. Put $\lambda_{0}=\operatorname{card}(H)$, i.e. $H=\left\{h_{\beta} ; \beta<\lambda_{0}\right\}$. By induction, define $\lambda_{n+1}=\lambda_{n}^{+}, n<\aleph_{0}$. Put $\lambda=\sup _{n<\aleph_{0}} \lambda_{n}$. Then $\lambda$ has cofinality $\omega$. By 3.16, $\operatorname{Ext}_{R}\left(R / J, M^{\lambda} / M_{\lambda}\right)=0$. Since $R / J$ is a cyclic non-projective module, $M^{\lambda} / M_{\lambda}$ is injective, and $\operatorname{Ext}_{R}\left(R / I, M^{\lambda} / M_{\lambda}\right)=0$. For each $\nu<\lambda$, denote by $\pi_{\nu}$ the projection of $M^{\lambda}$ onto $M$. Define $f \in \operatorname{Hom}_{R}\left(I, M^{\lambda} / M_{\lambda}\right)$ by $f\left(x_{\alpha}\right)=m_{\alpha}+M_{\lambda}, \alpha<\kappa$, where $m_{\alpha} \in M^{\lambda}$ is defined by

$\pi_{\nu}\left(m_{\alpha}\right)=h_{\nu}\left(x_{\alpha}\right)$ provided $\nu<\lambda_{0}$

$\pi_{\nu}\left(m_{\alpha}\right)=h_{\beta}\left(x_{\alpha}\right)$ provided $\nu=\lambda_{n}+\beta, \beta<\lambda_{0}, n<\aleph_{0}$;

$\pi_{\nu}\left(m_{\alpha}\right)=0$ otherwise.

Since $\operatorname{Ext}_{R}\left(R / I, M^{\lambda} / M_{\lambda}\right)=0$, there is a $g \in \operatorname{Hom}_{R}\left(R, M^{\lambda} / M_{\lambda}\right)$ such that $g\lceil I=$ $f$. Hence, there is some $y \in M^{\lambda}$ such that $y . x_{\alpha}-m_{\alpha} \in M_{\lambda}$, for all $\alpha<\kappa$. For $n<\aleph_{0}$, put $A_{n}=\left\{\alpha<\kappa ; y \cdot x_{\alpha}-m_{\alpha} \in M_{n}\right\}$. Then $A_{n} \subseteq A_{n+1}$ for all $n<\aleph_{0}$, and $\kappa=\bigcup_{n<\aleph_{0}} A_{n}$. Clearly, there is a $p<\aleph_{0}$ such that $\operatorname{card}\left(A_{p}\right)>\aleph_{0}$. Then $\pi_{\nu}\left(y \cdot x_{\alpha}-m_{\alpha}\right)=0$ for all $\lambda_{p} \leq \nu<\lambda$ and all $\alpha \in A_{p}$.

Put $K=\bigoplus \sum_{\alpha \in A_{p}} x_{\alpha} R$. We shall prove that $\operatorname{Ext}_{R}(R / K, M)=0$, i.e. that any $h \in \operatorname{Hom}_{R}(K, M)$ extends into some $h^{\prime} \in \operatorname{Hom}_{R}(R, M)$. First, there is some 
$\beta<\lambda_{0}$ such that $h_{\beta}\left\lceil K=h\right.$. Put $\nu_{0}=\lambda_{p}+\beta$. Then $h\left(x_{\alpha}\right)=\pi_{\nu_{0}}\left(m_{\alpha}\right)=\pi_{\nu_{0}}\left(y \cdot x_{\alpha}\right)$ for all $\alpha \in A_{p}$. Define $h^{\prime} \in \operatorname{Hom}_{R}(R, M)$ by $h^{\prime}(1)=\pi_{\nu_{0}}(y)$. Then $h^{\prime} \uparrow K=h$, and $\operatorname{Ext}_{R}(R / K, M)=0$. Since $R / K$ is a cyclic non-projective module, we infer that $M$ is injective, a contradiction.

We turn to the case when $\mathcal{I} \mathcal{T}$ contains all countably generated non-projective modules:

Theorem 4.3. Let $R$ be an $\aleph_{0}$-saturated ring. Then either

(i) $R$ is right artinian, or

(ii) $R$ is von Neumann regular and each right ideal of $R$ is countably generated.

Proof. Assume that $R$ is not von Neumann regular. Proving indirectly, we show that $R$ is right perfect: otherwise, there exist elements $a_{i} \in R, i<\aleph_{0}$, such that $\left(R a_{i} \ldots a_{0} ; i<\aleph_{0}\right)$ is a strictly decreasing chain of principal left ideals of $R$. Let $1_{i}, i<\aleph_{0}$, be the canonical basis of the free module $F=R^{\left(\aleph_{0}\right)}$ and let $G=$ $\sum_{i<\aleph_{0}}\left(1_{i}-1_{i+1} \cdot a_{i}\right) R \subseteq F$. Put $M=F / G$. By Bass' lemma, $M$ is a countably generated flat module, but $M$ is not projective. Since $R$ is not von Neumann regular, there exists a non-flat left $R$-module $N$. Let $C$ be an injective cogenerator for $M o d-\mathbb{Z}$. Since $\operatorname{Tor}_{R}(M, N)=0$, we have ([CaEi,IV,Proposition 5.1])

$$
\operatorname{Ext}_{R}\left(M, \operatorname{Hom}_{\mathbb{Z}}(N, C)\right) \simeq \operatorname{Hom}_{\mathbb{Z}}\left(\operatorname{Tor}_{R}(M, N), C\right)=0 .
$$

Since $N$ is not flat in $R$-Mod and $C$ is a cogenerator for $M o d-\mathbb{Z}, H_{0} m_{\mathbb{Z}}(N, C)$ is not injective. Hence, $M$ is not i-test, a contradiction. Therefore, $R$ is right perfect and right noetherian, by 4.2. Thus, $R$ is right artinian, and (i) holds.

If $R$ is von Neumann regular, then 4.2 gives (ii).

Now, we pause to present the "rare" examples of rings possessing many test modules. The first one is an artinian non-singular ring such that $R$ is fully saturated:

Example 4.4. Let $K$ be a skew-field. Denote by $R=U T_{2}(K)$ the ring of all upper triangular $2 \times 2$ matrices over $K$. Then $R$ is a (left and right) artinian and (left and right) non-singular ring, and $R$ is fully saturated.

Proof. $R$ is well-known to be artinian and hereditary. Denote by $e$ and $f$ the orthogonal idempotents of $R$ such that $e_{00}=f_{11}=1$, and all other entries in $e$ and $f$ are zero. Then $J_{0}=e R / \operatorname{Soc}(e R)$ and $J_{1}=f R$ are - up to isomorphism- the only simple modules. Moreover, $J_{0}$ is injective, and $e R \simeq I\left(J_{1}\right)$. Let $M$ be any module. There exist cardinals $\kappa$ and $\lambda$ such that $S o c(M) \simeq J_{0}^{(\kappa)} \oplus J_{1}^{(\lambda)}$. Since $J_{0}^{(\kappa)}$ is injective, there are submodules $N$ and $P$ in $M$ such that $M=N \oplus P, P \simeq J_{0}^{(\kappa)}$ and $\operatorname{Soc}(N) \simeq J_{1}^{(\lambda)}$. Then $I(N) \simeq(e R)^{(\lambda)}$ is projective, and so is $N$. Since $\{e, f\}$ is a complete basic set of idempotents of $R$, there are cardinals $\mu$ and $\nu$ such that $N \simeq J_{1}^{(\mu)} \oplus(e R)^{(\nu)}$. Hence, $M$ is isomorphic to a direct sum of direct powers of the modules $J_{0}, J_{1}$ and $e R$. If $M$ is non-projective and non-injective, then the direct power of $J_{0}$, and of $J_{1}$, respectively, is non-zero in this decomposition. Since $\operatorname{Ext}_{R}\left(J_{0}, J_{1}\right) \neq 0$, the assertion holds true.

Our second example is again a fully saturated ring:

Example 4.5. Let $R$ be a commutative local artinian principal ideal ring. Then $R$ is fully saturated. 
Proof. It is well-known that each module is a direct sum of cyclic modules, and the ideals of $R$ form a chain

$$
0=x^{m} R \subset x^{m-1} R \subset \cdots \subset x R=\operatorname{Rad}(R) \subset R,
$$

where $x$ is a generator of $\operatorname{Rad}(R)$ (see [FuSa]). Since $\operatorname{Soc}(R)$ is simple, $R$ is a QF-ring.

Hence, we have to prove that $\operatorname{Ext}_{R}\left(R / x^{i} R, R / x^{j} R\right) \neq 0$ for all $0<i, j<m$. Define $f \in H^{\prime o m}{ }_{R}\left(x^{i} R, R / x^{j} R\right)$ by $f\left(x^{i}\right)=1+x^{j} R$ provided $i+j \leq m$, and by $f\left(x^{i}\right)=x^{i+j-m}+x^{j} R$ otherwise. Then $f \neq g\left\lceil x^{i} R\right.$, for all $g \in \operatorname{Hom}_{R}\left(R, R / x^{j} R\right)$. This proves that $\operatorname{Ext}_{R}\left(R / x^{i} R, R / x^{j} R\right) \neq 0$.

The ring $R$ from the previous example is an artinian valuation ring. Also noetherian valuation domains possess many i-test modules:

Example 4.6. Let $R$ be a noetherian valuation domain which is not a field. Then $R$ is $n$-saturated for each $n<\aleph_{0}$, but it is not $\aleph_{0}$-saturated.

Proof. Since $R$ is an almost maximal valuation domain, each finitely generated module is a direct sum of cyclic modules ([FuSa $]$ ). Hence, it suffices to prove that each cyclic non-projective module is i-test. By the premise, the ideals of $R$ form a chain

$$
0=\bigcap_{n<\aleph_{0}} x^{n} R \subset \cdots \subset x^{n+1} R \subset x^{n} R \subset \cdots \subset x^{2} R \subset x R=\operatorname{Rad}(R) \subset R .
$$

Let $N$ be a module. Assume there is some $0<n<\aleph_{0}$ such that $\operatorname{Ext}_{R}\left(R / x^{n} R, N\right)=$ 0 . Since $\operatorname{Ann}\left(x^{n}\right)=0$, each element of $N$ is divisible by $x^{n}$. Then each element of $N$ is divisible by $x^{m}$ and $\operatorname{Ext}_{R}\left(R / x^{m} R, N\right)=0$, for all $0<m<\aleph_{0}$. By Baer's Criterion, $N$ is injective. Since $R$ is not right artinian, the last assertion follows from 4.3.

Note that 4.6 shows that 4.2 and 4.3 apply to different classes of rings. Another example of this fact is

Example 4.7. Let $K$ be a universal differential field of characteristic 0 with differentiation $D$ (i.e. $\operatorname{char}(K)=0$; for each $n<\aleph_{0}$, each polynomial equation in indeterminates $x_{0}=x, x_{1}=D(x), \ldots, x_{n-1}=D^{n-1}(x)$ has a solution in $K$; and each homogenous linear $D$-differential equation has a non-trivial solution in $K$ ). Denote by $R=K[y, D]$ the ring of all differential polynomials in one indeterminate $y$ over $K$ (i.e. the elements of $R$ are polynomials from $K[y]$ with usual addition, and with multiplication given by the identity $y a=a y+D(a)$ and its consequences). Then $R$ is $n$-saturated for each $n<\aleph_{0}$, but it is not $\aleph_{0}$-saturated.

Proof. We shall need several well-known properties of $R$ (proved in [Fa], [CzFa] and $[\mathrm{K}])$ : first, $R$ is a simple non-commutative principal right ideal domain. Moreover, all simple modules are isomorphic to a simple module $J$, and $J$ is injective. Let $K$ be a right ideal of $R$. Since $R$ has a right division algorithm, $R / K$ is a semisimple module. Let $Q$ be the right skew field of quotients of $R$. Then $Q$ is an injective module. Since $R$ is right noetherian, each injective module is isomorphic to a direct sum of copies of $Q$ and $J$. In particular $I(N) / N$ is isomorphic to a direct power of $J$ for each module $N$. If $F$ is a finitely generated module with $\operatorname{Soc}(F)=0$, then $F$ is flat, whence $F$ is free. 
Now, we prove that each finitely generated non-projective module $F$ is i-test: Assume $\operatorname{Ext}_{R}(F, M)=0$ for a module $M$. Note that $F=\operatorname{Soc}(F) \oplus G$, where $G$ is finitely generated and $\operatorname{Soc}(G)=0$. Hence $G$ is free, and $\operatorname{Soc}(F) \neq 0$. Similarly, $M=\operatorname{Soc}(M) \oplus N$, where $\operatorname{Soc}(N)=0$ and $I(N) \simeq Q^{(\kappa)}$ for a cardinal $\kappa$. W.l.o.g., we can assume that $N \neq 0$. We have $\operatorname{Ext}_{R}(J, N)=0$ and $\operatorname{Hom}_{R}\left(J, Q^{(\kappa)}\right)=0$. Then also $\operatorname{Hom}_{R}(J, I(N) / N)=0$. Since $I(N) / N$ is isomorphic to a direct power of $J$, we infer that $N=I(N)$.

The last assertion is a consequence of 4.3 .

In fact, the proof of the last assertions of 4.6 and 4.7 is constructive. Taking the module $M=F / G$ as in the proof of 4.3 , we obtain a particular countably generated non-projective module which is not i-test.

Now, we proceed with the structure theory and show that the "rare" examples of 4.4-4.7 are in a sense typical. First, we have

Lemma 4.8. Let $R$ be a 1-saturated ring. Then all non-projective simple modules are isomorphic.

Proof. Let $J$ be a non-projective simple module and $N=N_{0}$ be a non-injective module. By the premise, $\operatorname{Ext}_{R}\left(J, N_{0}\right) \neq 0$, so $\operatorname{Hom}_{R}\left(J, I(N) / N_{0}\right) \neq 0$. Hence, there is a module $N_{1}$ such that $N_{0} \subset N_{1} \subseteq I(N)$ and $N_{1} / N_{0} \simeq J$. If $N_{1} \neq I(N)$, then $\operatorname{Ext}_{R}\left(J, N_{1}\right) \neq 0$, and $\operatorname{Hom}_{R}\left(J, I(N) / N_{1}\right) \neq 0$. Proceeding similarly, we see that the module $I(N) / N$ has a (transfinite) composition series with factors isomorphic to $J$. If $J^{\prime}$ is another non-projective simple module, then $\operatorname{Ext}_{R}\left(J^{\prime}, N\right) \neq$ 0 , and $H_{R}\left(J^{\prime}, I(N) / N\right) \neq 0$, whence $J^{\prime} \simeq J$.

The following theorem shows that we can restrict our investigation to indecomposable rings:

Theorem 4.9. Let $\kappa$ be a cardinal. Let $R$ be a $\kappa$-saturated (fully saturated) ring. Then either

(i) $R$ is an indecomposable ring; or

(ii) $R=R^{\prime} \boxplus R^{\prime \prime}$, where $R^{\prime \prime}$ is a semisimple ring and $R^{\prime}$ is an indecomposable ring such that $R^{\prime}$ is $\kappa$-saturated (fully saturated).

On the other hand, if $R=R^{\prime} \boxplus R^{\prime \prime}$ and $R^{\prime}, R^{\prime \prime}$ are as in (ii), then $R$ is $\kappa$-saturated (fully saturated).

Proof. First, note that for any decomposition $R^{\prime} \boxplus R^{\prime \prime}$ of the ring $R$, either $R^{\prime}$ or $R^{\prime \prime}$ is semisimple. Indeed, taking any non-projective simple right $R^{\prime}$-module $M$ and any non-injective right $R^{\prime \prime}$-module $N$, we have $\operatorname{Ext}_{R}(M, N)=0$, a contradiction. Let $B$ be a representative set of all projective simple modules (possibly, $B=\emptyset$ ). Let $J$ be a simple non-projective module. By 4.8, $A=B \cup\{J\}$ is a representative set of all simple modules. Let $C$ and $D$ be two disjoint subsets of $A$. Denote by $I_{C}$ and $I_{D}$ the trace of $C$ and of $D$, respectively, in $R$. Then $I_{C}$ and $I_{D}$ are two-sided ideals of $R$. Moreover, $\operatorname{Hom}_{R}\left(I_{C}, I_{D}\right)=0$, and $\operatorname{Ext}_{R}\left(R / I_{C}, I_{D}\right)=0$. Hence, either $I_{C}$ is a summand of $R$, or $I_{D}$ is injective (and a summand of $R$ ).

Assume $C$ and $D$ are two infinite disjoint subsets of $A$. Then neither $I_{C}$ nor $I_{D}$ is finitely generated, a contradiction. Hence, $A$ is finite. This implies that either $R$ is indecomposable, or $R$ has a decomposition $R=R^{\prime} \boxplus R^{\prime \prime}$, where $R^{\prime \prime}$ is semisimple and $R^{\prime}$ is indecomposable. The final assertion follows from the fact that

$$
\operatorname{Ext}_{R}(M, N) \simeq \operatorname{Ext}_{R^{\prime}}\left(M R^{\prime}, N R^{\prime}\right) \oplus \operatorname{Ext}_{R^{\prime \prime}}\left(M R^{\prime \prime}, N R^{\prime \prime}\right)
$$

whenever $M, N \in M o d-R$. 
As we shall see in Sections 5 and 6, an important distinction between indecomposable 1-saturated rings comes from the number of isomorphism classes of simple modules. This leads to the following definition:

Definition 4.10. Let $R$ be a non-semisimple indecomposable ring such that $R$ is 1-saturated. Then $R$ is said to be of type $I$ provided that all simple modules are isomorphic. Assume $R$ is of type I. Then

(i) $R$ is of type Ia provided $\operatorname{Sing}(R) \neq 0$,

(ii) $R$ is of type $I b$ provided $\operatorname{Sing}(R)=0$ and $\operatorname{Rad}(R)=0$,

(iii) $R$ is of type $I c$ provided $\operatorname{Sing}(R)=0$ and $\operatorname{Rad}(R) \neq 0$.

Further, $R$ is said to be of type $I I$ provided that there are at least two non-isomorphic simple modules.

Note that all the types from 4.10 do occur: Ia in Example 4.5, Ib in 4.7, Ic in 4.6, and II in 4.4.

Proposition 4.11. Let $R$ be a non-semisimple indecomposable ring such that $R$ is 1-saturated. Clearly, either $R$ is of type I or of type II. Moreover,

(i) if $R$ is of type Ia, then $R$ is isomorphic to a full matrix ring over a local right artinian ring,

(ii) if $R$ is of type $I b$, then $R$ is a simple ring such that each right ideal is countably generated,

(iii) if $R$ is of type $I c$, then $R$ is right noetherian with $\operatorname{Soc}(R)=0$.

If $R$ is of type II, then $R$ is right semiartinian and right hereditary and, up to isomorphism, there exist exactly two simple modules. One of them, $J$, is $\Sigma$-injective and non-projective, and the other, $P$, is projective.

Proof. Take a simple non-projective module $J$. If $B$ is a representative set of all simple projective modules, then $A=B \cup\{J\}$ is a representative set of all simple modules by 4.8. For $C \subseteq A$, denote by $I_{C}$ the trace of $C$ in $R$. We distinguish the following four cases:

(1a) $B=\emptyset$ and $I_{J} \neq 0$. Then $R$ is not von Neumann regular, and $R$ is right noetherian by 4.2. Since $J$ embeds into $R$, we have $\operatorname{Soc}(R)=I_{J} \neq 0$. Moreover, if $\operatorname{Soc}(R / \operatorname{Soc}(R))=0$, then $\operatorname{Hom}_{R}\left(I_{J}, N\right)=0$ for any submodule $N$ of $R / \operatorname{Soc}(R)$. Hence, $\operatorname{Ext}_{R}\left(R / I_{J}, N\right)=0, N$ is injective, and $R / \operatorname{Soc}(R)$ is completely reducible, a contradiction. Similarly, it follows that $R$ has a (finite) socle sequence, and $R$ is right artinian. This implies that $R \simeq(e R)^{(n)}$ for some $n<\aleph_{0}$ and some indecomposable idempotent $e \in R$. Then $R \simeq \operatorname{Hom}_{R}\left((e R)^{(n)},(e R)^{(n)}\right) \simeq M_{n}(e R e)$, where $e R e$ is a local right artinian ring.

(1b) $B=\emptyset, I_{J}=0$ and $\operatorname{Rad}(R)=0$. Let $I \neq R$ be a two-sided ideal of $R$. Let $M$ be a maximal right ideal containing $I$. Then $J \simeq R / M$ and $I . J=0$. Since $\operatorname{Rad}(R)=0$, we have $\operatorname{Ann}(J)=0$ and $I=0$. Hence, $R$ is a simple ring.

(1c) $B=\emptyset, I_{J}=0$ and $\operatorname{Rad}(R) \neq 0$. By $4.2, R$ is right noetherian. Also $\operatorname{Soc}(R)=I_{J}=0$. Further, assume $\operatorname{Sing}(R) \neq 0$. Take $0 \neq r \in R$ such that $K=$ $\operatorname{Ann}(r) \unlhd R$. Then $R / K \simeq r R$ is a submodule of $I(K) / K$. Since $\operatorname{Ext}_{R}(J, K) \neq$ 0 , also $\operatorname{Hom}_{R}(J, I(K) / K) \neq 0$, and $I(K) / K$ has a transfinite composition series with factors isomorphic to $J$. In particular, $J$ embeds into $R / K \simeq r R \subset R$, a contradiction. Hence, $R$ is right non-singular. 
(2) $B \neq \emptyset$. Then $I_{B} \neq 0$. Since $\operatorname{Hom}_{R}\left(I_{B}, I_{J}\right)=0$ and $R$ is indecomposable, we infer that $\operatorname{Ext}_{R}\left(R / I_{B}, I_{J}\right)=0$ and $I_{J}=0$. Take $P \in B$ and put $C=B \backslash\{P\}$. Similarly, we get $I_{C}=0$, i.e. $C=\emptyset$ and $A=\{P, J\}$. Take a cardinal $\kappa$ and consider the module $N=J^{(\kappa)}$. Since $\operatorname{Hom}_{R}\left(I_{P}, N\right)=0$, we have $\operatorname{Ext}_{R}\left(R / I_{P}, N\right)=0$, and $N$ is injective.

Let $M$ be an arbitrary module. Denote by $M_{P}$ the trace of $P$ in $M$. Let $N$ be a submodule of $M / M_{P}$. Since $\operatorname{Hom}_{R}\left(I_{P}, N\right)=0$, we have $\operatorname{Ext}_{R}\left(R / I_{P}, N\right)=0, N$ is injective and $M / M_{P}$ is semisimple. Hence, $M / M_{P}$ is isomorphic to a direct power of $J$. In particular, $R$ is right semiartinian.

Proving indirectly, assume $R$ is not right hereditary. Then there are an injective module $I$ and a submodule $K$ of $I$ such that $N=I / K$ is not injective. Put $M=R / I_{P}$. Since the sequence $0 \rightarrow I_{P} \rightarrow R \rightarrow M \rightarrow 0$ is exact and $I_{P}$ is projective, we get $0=\operatorname{Ext}_{R}\left(I_{P}, K\right) \rightarrow \operatorname{Ext}_{R}^{2}(M, K) \rightarrow \operatorname{Ext}_{R}^{2}(R, K)=0$, and $\operatorname{Ext}_{R}^{2}(M, K)=0$. Since the sequence $0 \rightarrow K \rightarrow I \rightarrow N \rightarrow 0$ is exact, we have $0=\operatorname{Ext}_{R}(M, I) \rightarrow \operatorname{Ext}_{R}(M, N) \rightarrow \operatorname{Ext}_{R}^{2}(M, K)=0$, and $\operatorname{Ext}_{R}(M, N)=0$. Then $M$ is not i-test, a contradiction.

Now, it is clear that $R$ is of type Ia (Ib, Ic, II) iff the case 1a (1b, 1c, 2) applies to $R$, and the assertion follows.

\section{A MATRIX CHARACTERIZATION FOR TYPE II}

In this section, using more involved arguments, we shall obtain a full characterization of rings of type II as certain generalized upper triangular matrix rings (Theorem 5.14). Moreover, in Theorem 5.16, we prove that 2-saturated rings of type II are exactly the rings Morita equivalent to the matrix rings from Example 4.4 .

As a first step of the characterization, we have

Lemma 5.1. Any ring of type II is right artinian.

Proof. Since $R$ is right semiartinian and 4.2 holds, it suffices to prove that $R$ is not von Neumann regular. On the contrary, assume $R$ is von Neumann regular. By 4.11, $R / I_{P} \simeq J^{(n)}$ for some $n<\aleph_{0}$. Hence, $\bar{R}=R / I_{P}$ is a simple artinian ring and there is a complete set of orthogonal idempotents $\left\{\bar{e}_{0}, \ldots, \bar{e}_{n-1}\right\} \subseteq \bar{R}$ such that $\bar{e}_{i} \bar{R}$ is a minimal right ideal of $\bar{R}$ for all $i<n$. Since $R$ is von Neumann regular, this set can be lifted modulo $I_{P}$ into a complete set of orthogonal idempotents, $\left\{e_{0}, \ldots, e_{n-1}\right\}$, of the ring $R$ so that $e_{i}+I_{P}=\bar{e}_{i}$ for all $i<n$. Since $R$ is not semisimple, $\operatorname{dim}(\operatorname{Soc}(R))=\operatorname{dim}\left(I_{P}\right)=\kappa$ for some $\kappa \geq \aleph_{0}$. Hence, $\operatorname{Soc}(R)=\bigoplus \sum_{i<n} \operatorname{Soc}\left(e_{i} R\right)$ and there is some $e=e_{i}$ such that $\operatorname{dim}(\operatorname{Soc}(e R))=\kappa$. Since $R$ is von Neumann regular, there are a complete decomposition $\bigoplus \sum_{\alpha<\kappa} s_{\alpha} R$ of $\operatorname{Soc}(R)$ and a subset $A \subseteq \kappa$ such that

(1) $0 \in A, \operatorname{card}(A)=\kappa$,

(2) $f=s_{0}$ is an idempotent of $R$ such that efe $=f$,

(3) $\operatorname{Soc}((e-f) R)=\bigoplus \sum_{\alpha \in A, \alpha \neq 0} s_{\alpha} R$, and

(4) $\operatorname{Soc}((1-e) R)=\bigoplus \sum_{\alpha \notin A} s_{\alpha} R$.

By $4.11, \operatorname{Soc}(R) \unlhd R$ and $s_{\alpha} R \simeq P$ for all $\alpha<\kappa$. Hence, there is a canonical ring isomorphism $\phi: \operatorname{End}_{R}(\operatorname{Soc}(R)) \simeq C F M_{\kappa}(K)$, where $K=\operatorname{End}_{R}(P)$. Since $R$ is right non-singular, the canonical mapping $\varphi: \operatorname{End}_{R}(I(R)) \rightarrow \operatorname{End}_{R}(\operatorname{Soc}(R))$ defined by $\varphi(x)=x \uparrow \operatorname{Soc}(R)$ is a ring isomorphism (see e.g. [L, $\S 4.3$ and $\S 4.5]$ ). Denote by $Q$ the maximal right quotient ring of $R$. Then $Q=I(R)$ (as modules), 
and the canonical mapping $\psi: Q \rightarrow \operatorname{End}_{R}(I(R))$ given by $h(q)(1)=(1) q$ is a ring isomorphism.

W.l.o.g., we can view $R$ as a submodule of $Q$. Moreover, since $\pi=\phi \varphi \psi$ is a ring isomorphism, we can identify $Q$ with $C F M_{\kappa}(K)$, whence $R$ becomes a subring of $C F M_{\kappa}(K)$. By (2), (3) and (4), $m=\pi(e)$ is a matrix such that $m_{\alpha \alpha}=1$ provided $\alpha \in A$, and $m_{\alpha \beta}=0$ otherwise. If $s \in \operatorname{Soc}(R)$, then $\psi(q s)(1)=(1)(q s)=((1) q) s=$ $(s) q=s^{\prime} \in \operatorname{Soc}(R)$, whence $\psi(q s)=\psi\left(s^{\prime}\right)$ for all $q \in Q$. Thus, $\operatorname{Soc}(R)$ is a left ideal of the ring $C F M_{\kappa}(K)$. Moreover, if $s \in \operatorname{Soc}(R), s=\sum_{\alpha \in F} s_{\alpha} r_{\alpha}$ for a finite set $F \subset \kappa$, then $\pi(s)$ is a matrix which is zero in any row indexed by $\alpha \in \kappa \backslash F$. Since $\pi(s)$ is also column finite, it has only finitely many non-zero entries. Further, by $(2),(\pi(f))_{00}=1$ and $(\pi(f))_{\alpha \beta}=0$ otherwise. Define $\left\{f_{n}, n<\aleph_{0}\right\} \subset Q$ by $f_{0}=f$, and $\left(f_{n}\right)_{n 0}=1,\left(f_{n}\right)_{\alpha \beta}=0$ otherwise, for $0<n<\aleph_{0}$. Since $\operatorname{Soc}(R)$ is a left ideal of $Q$, we infer that $f_{n} \in \operatorname{Soc}(R)$ for all $n<\aleph_{0}$.

We shall construct a cyclic non-projective module $M$ which is not i-test: Consider the right ideal $I=\bigoplus \sum_{n<\aleph_{0}, n \text { even }}\left(f_{n}-f_{n+1}\right) R$. Since $I$ is not finitely generated, $M=R / I$ is not projective.

It remains to construct a non-injective module $N$ such that $\operatorname{Ext}_{R}(M, N)=$ 0 . Let $\lambda=\operatorname{card}((e R e+\operatorname{Soc}(R)) / \operatorname{Soc}(R))$. Then $(e \operatorname{Re}+\operatorname{Soc}(R)) / \operatorname{Soc}(R)=$ $\left\{r_{\beta}+\operatorname{Soc}(R), \beta<\lambda\right\}$ for some $r_{\beta} \in e R e, \beta<\lambda$, and $r_{0}=0$. Put $L=$ $Q^{(\lambda)}$ and denote by $\pi_{\beta}, \beta<\lambda$, the $\beta$-th projection of $L$ onto $Q$. Note that $\operatorname{Soc}(L)=(\operatorname{Soc}(R))^{(\lambda)} \unlhd L \unlhd I(L)$, and $I(L) / \operatorname{Soc}(L)$ is a completely reducible module. W.l.o.g., we shall view $Q$ as a submodule of $L$ consisting of all $l \in L$ such that $\pi_{\beta}(l)=0$ for all $0<\beta<\lambda$. Consider the matrix $q \in Q$ defined by $q_{i, i+1}=q_{i+1, i}=1$ provided $i<\aleph_{0}, i$ even, and by $q_{\alpha \beta}=0$ otherwise. Note that $q=e q e$ and $e+q \notin S o c(R)$, as $e+q$ has infinitely many nonzero entries. Moreover, take $r \in R$ such that $(e+q) r \in \operatorname{Soc}(R)$. Then $(e+q)$ ere $\in \operatorname{Soc}(R)$. Assume $(e R e) r \nsubseteq \operatorname{Soc}(R)$. Then $\left(e r^{\prime} e\right)(e r e)=e+s$, for some $r^{\prime} \in R$ and $s \in \operatorname{Soc}(R)$. Since $\bar{e} \bar{R} \bar{e} \simeq \operatorname{End}_{\bar{R}}(\bar{e} \bar{R}) \simeq \operatorname{End}_{R}(J)$ is a skew-field, also $($ ere $)\left(e r^{\prime} e\right)=e+s^{\prime}$ for some $s^{\prime} \in \operatorname{Soc}(R)$, whence $e+q=(e+q)\left(\operatorname{erer}^{\prime} e-s^{\prime}\right) \in \operatorname{Soc}(R)$, a contradiction. This implies that there exists a maximal submodule $N$ of $I(L)$ such that $\operatorname{Soc}(L) \subseteq N$, $e \notin N$, and $q_{\beta} \in N$ for all $0<\beta<\lambda$. Here, $q_{\beta}$ denotes the element of $L$ defined by $\pi_{0}\left(q_{\beta}\right)=r_{\beta}, \pi_{\beta}\left(r_{\beta}\right)=e+q$, and $\pi_{\beta^{\prime}}\left(r_{\beta}\right)=0$ otherwise. Since $N \neq I(L)$ and $\operatorname{Soc}(L) \unlhd N \unlhd I(L), N$ is not injective.

Finally, let $\phi \in \operatorname{Hom}_{R}(I, N)$. Then there is some $x \in I(L)$ with $x e\left(f_{n}-f_{n+1}\right)=$ $\phi\left(f_{n}-f_{n+1}\right)$ for all $n<\aleph_{0}$. Since $N$ is a maximal submodule of $I(L)$, we have $e R+N=I(L)$, and $x=e r+y$ for some $r \in R$ and $y \in N$. Then ere $=r_{\beta}$ for some $\beta<\lambda$. If $r_{\beta} \neq 0$ (i.e. if $\beta>0$ ), then $r_{\beta}\left(f_{n}-f_{n+1}\right)=q_{\beta}\left(f_{n}-f_{n+1}\right)$, whence $\left(q_{\beta}-y e\right)\left(f_{n}-f_{n+1}\right)=x e\left(f_{n}-f_{n+1}\right)=\phi\left(f_{n}-f_{n+1}\right)$. Define $\varphi \in \operatorname{Hom}_{R}(R, N)$ by $\varphi(1)=q_{\beta}+$ ye provided $\beta>0$, and by $\varphi(1)=y e$ otherwise. Then $\varphi \uparrow I=\phi$, and $\operatorname{Ext}_{R}(R / I, N)=0$.

Now, we introduce a class of rings which plays crucial role in characterizing rings of type II:

Definition 5.2. Let $0<m<\aleph_{0}$. Let $S$ and $T$ be skew-fields such that $T$ is a subring of $M_{m}(S)$. Denote by - the mapping from $M_{m+1}(S)$ to $M_{m}(S)$ defined by $\left(a^{-}\right)_{i j}=a_{i j}$ for all $a \in M_{m+1}(S)$ and $i, j<m$. Define $R=U T(m, S, T)$ as the subring of $M_{m+1}(S)$ consisting of all matrices $a \in M_{m+1}(S)$ satisfying

(1) $a_{m i}=0$ for all $i<m$, and

(2) $a^{-} \in T$. 
Note that the rings $U T(m, S, T)$ include following important particular cases:

(1) upper triangular matrix rings of degree two over skew-fields (as $U T(1, K, K)$ $=U T_{2}(K)$ for any skew-field $\left.K\right)$-see Example 4.4;

(2) the rings $U T(1, S, T)$, where $T \subset S$ are skew-fields and $S$ is a quadratic extension of $T$ (these examples are essential for several recent applications, see e.g. [CT1], [CT2], and [Si]);

(3) the ring $U T(2, \mathbb{C}, \varphi(\mathbb{H}))$, where $\mathbb{C}$ is the field of all complex numbers, $\mathbb{H}$ the skew-field of all quaternions and $\varphi$ the canonical ring embedding of $\mathbb{H}$ into $M_{2}(\mathbb{C})$.

Basic properties of the rings $U T(m, S, T)$ can easily be described:

Lemma 5.3. Let $R=U T(m, S, T)$. For each $i \leq m$, denote by $e_{i}$ the matrix from $R$ defined by $\left(e_{i}\right)_{i m}=1$ and $\left(e_{i}\right)_{j k}=0$ otherwise. Put $e=e_{m}, f=1-e, P=e R$ and $J=R / \operatorname{Soc}(R)$.

(i) If $I$ is a proper right ideal of $R$, then either $I=f R$ or $I \subseteq \bigoplus \sum_{i \leq m} e_{i} R$.

(ii) $\operatorname{Soc}(R)=\bigoplus \sum_{i \leq m} e_{i} R$ and $\operatorname{Rad}(R)=\bigoplus \sum_{i<m} e_{i} R$.

(iii) The mapping $\psi: R / \operatorname{Soc}(R) \rightarrow T$ defined by $\psi(r+\operatorname{Soc}(R))=r^{-}$is a ring isomorphism.

(iv) $R$ is an indecomposable right hereditary right artinian basic ring. The set $\{e, f\}$ is a complete basic set of idempotents of $R$.

(v) $\{P, J\}$ is a representative set of all simple modules. Moreover, $P$ is projective, but not injective, while $J$ is $\sum$-injective, but not projective.

(vi) $I(R)=M_{m+1}(S)$, and the maximal right quotient ring of $R$ is isomorphic to $M_{m+1}(S)$.

Proof. By easy matrix computations.

As further step of the characterization, we have

Theorem 5.4. Any ring of type II is Morita equivalent to some $U T(m, S, T)$.

Proof. Let $R$ be a ring of type (II). By 4.11, we have $0 \subset \operatorname{Rad}(R) \subset \operatorname{Soc}(R)=I_{P}$, where $P$ is (up to isomorphism) the only projective module. Moreover, by 5.1 , there is a complete orthogonal set, $\left\{e_{0}, \ldots, e_{k}, e_{0}^{\prime}, \ldots, e_{l}^{\prime}\right\}$, of primitive idempotents of $R$ such that $P \cong e_{i} R$ for all $i \leq k$ and $e_{i}^{\prime} R \cong e_{i^{\prime}}^{\prime} R$ for all $i, i^{\prime} \leq l$. By 4.11, $\operatorname{Soc}(R) \cong P^{(n)}$ for some $k<n<\aleph_{0}$, and $R / \operatorname{Soc}(R)$ is a simple artinian ring. Put $S=\operatorname{End}_{R}(P)$. Then $S$ is a skew-field. Let $R^{\prime}$ be the basic ring of $R$. Since $R$ is Morita equivalent to $R^{\prime}$, it suffices to show that $R^{\prime}$ is isomorphic to some $U T(m, S, T)$.

Clearly, $R^{\prime}=(e+f) R(e+f)$, where $e=e_{0}$ and $f=e_{0}^{\prime}$, and $\{e, f\}$ is a basic set of primitive idempotents of $R^{\prime}$. Let $m=\operatorname{dim}\left(\operatorname{Soc}\left(f R^{\prime}\right)\right)$. The same argument as in the proof of 5.1 shows that $R^{\prime}$ is (canonically isomorphic to) a subring of the full matrix ring $Q=M_{m+1}(S)$ so that $e_{m m}=1$, and $e_{j j^{\prime}}=0$ otherwise. Moreover, as in the proof of 5.1, we see that $\operatorname{Soc}\left(R^{\prime}\right)$ is a left ideal of $Q$. In particular, each of the matrices $x_{i}, i \leq m$, defined by $\left(x_{i}\right)_{i m}=1$ and by $\left(x_{i}\right)_{j j^{\prime}}=0$ otherwise, belongs to $R^{\prime}$. Put $X=\left\{q \in Q ; q_{i j}=0\right.$ for all $i \leq m$ and $\left.j<m\right\}$. Then $X \subseteq \operatorname{Soc}\left(R^{\prime}\right)$. Since $f$ is a primitive idempotent, we have $\operatorname{Rad}\left(R^{\prime}\right)=\operatorname{Rad}\left(f R^{\prime}\right)=\operatorname{Soc}\left(f R^{\prime}\right)$. Hence, $x_{i} \in \operatorname{Rad}\left(R^{\prime}\right)$ for each $i<m$. Since $\operatorname{Rad}\left(R^{\prime}\right) . \operatorname{Soc}\left(R^{\prime}\right) \subseteq \operatorname{Rad}\left(\operatorname{Soc}\left(R^{\prime}\right)\right)=0$, we have $X=\operatorname{Soc}\left(R^{\prime}\right)$. In particular, for each $r \in R^{\prime}$ and each $i<m$, we have $x_{i} r \in \operatorname{Soc}\left(R^{\prime}\right)$, whence $r_{m i}=0$ for all $i<m$. If $q \in Q$, define $q^{-} \in M_{m}(S)$ as in 5.2. Let $T=\left\{q^{-} ; q \in R\right\}$. Then $T$ is a subring of $M_{m}(S)$ such that $T \cong R^{\prime} / \operatorname{Soc}\left(R^{\prime}\right) \cong$ $f R^{\prime} / \operatorname{Rad}\left(f R^{\prime}\right)$ is a skew-field. By $5.2, R^{\prime} \cong U T(m, S, T)$. 
Rings Morita equivalent to $U T(m, S, T)$ are completely characterized as certain generalized upper triangular matrix rings, as follows:

Definition 5.5. Let $0<m, n, p<\aleph_{0}$. Let $S$ and $T$ be skew-fields such that $T$ is a subring of $M_{m}(S)$. Denote by $G T(m, n, p, S, T)$ the subring of $M_{m . n+p}(S)$ consisting of all matrices of the form $\left(\begin{array}{ll}A & B \\ 0 & C\end{array}\right)$, where $A \in M_{n}(T) \subseteq M_{m . n}(S)$, $B \in M_{m . n \times p}(S), C \in M_{p}(S)$ and 0 is the zero matrix from $M_{p \times m . n}(S)$.

Theorem 5.6. Let $0<m<\aleph_{0}$. Let $S$ and $T$ be skew-fields such that $T$ is a subring of $M_{m}(S)$. Let $\bar{R}=U T(m, S, T)$. A ring $R$ is Morita equivalent to $\bar{R}$ if and only if there are $0<n, p<\aleph_{0}$ such that $R$ is isomorphic to $G T(m, n, p, S, T)$.

Proof. First, if $n=p$, then $R$ is (isomorphic to) the full matrix ring $M_{n}(\bar{R})$. Moreover, a ring $R^{\prime}$ is Morita equivalent to $\bar{R}$ iff there are $0<q<\aleph_{0}$ and an idempotent matrix $e \in M_{q}(\bar{R})$ such that $M_{q}(\bar{R}) e M_{q}(\bar{R})=M_{q}(\bar{R})$ and $R^{\prime} \cong e M_{q}(\bar{R}) e$. Using a suitable inner automorphism of $M_{q}(\bar{R})$, we can assume that $R^{\prime}$ is isomorphic to $e^{\prime} M_{q}(\bar{R}) e^{\prime}$, where $e^{\prime} \in M_{q}(\bar{R}) \subseteq M_{m . q+q}(S)$ is an idempotent matrix and there are subsets $\emptyset \neq A \subseteq q$ and $\emptyset \neq B \subseteq q$ such that $e_{j+m . i, j+m . i}^{\prime}=1$ for all $i \in A$ and $j<m, e_{m . q+i, m . q+i}^{\prime}=1$ for all $i \in B$, and $e_{i j}^{\prime}=0$ for all other pairs $i, j<m . q+q$. Finally, putting $n=\operatorname{card}(A)$ and $p=\operatorname{card}(B)$, we obtain a ring isomorphism $e^{\prime} M_{q}(\bar{R}) e^{\prime} \cong G T(m, n, p, S, T)$.

By 5.4 , it remains to determine which of the generalized upper triangular matrix rings $R$ defined in 5.5 really are of type II.

First, we show that if $m=1$ and $T=K$, then $R$ is of type II. This is a consequence of 4.4 and of the fact that the property " $R$ is $\lambda$-saturated" is Morita invariant for $\lambda \geq \aleph_{0}$ :

Lemma 5.7. Let $\lambda$ be an infinite cardinal. Let $R$ be a $\kappa$-saturated ring for all $\kappa<\lambda$ (a fully saturated ring). Let $\bar{R}$ be Morita equivalent to $R$. Then $\bar{R}$ is $\kappa$-saturated for all $\kappa<\lambda(\bar{R}$ is fully saturated $)$.

Proof. By 4.1, using the fact that the property "to be $\kappa$-generated" is Morita invariant for each $\kappa \geq \aleph_{0}$.

Example 5.8. Let $S$ be a skew-field. Let $R=G T(1, n, p, S, S)$ for some $0<n, p<$ $\aleph_{0}$, i.e. $R$ is Morita equivalent to $U T_{2}(S)$. Then $R$ is fully saturated. In particular, $R$ is of type $I I$.

Proof. By 4.4 and 5.7.

In order to decide the remaining cases, we need more information about basic properties of the generalized upper triangular matrix rings:

Lemma 5.9. Let $0<m, n, p<\aleph_{0}$. Let $T$ and $S$ be skew-fields such that $T$ is a subring of $M_{m}(S)$. Let $R=G T(m, n, p, S, T)$. For each $i<n$, define $f_{i} \in R$ by $\left(f_{i}\right)_{k k}=1$ provided $m . i \leq k<m .(i+1)$ and $\left(f_{i}\right)_{k l}=0$ otherwise. For each $j<p$, define $g_{j} \in R$ by $\left(g_{j}\right)_{k k}=1$ provided $k=m . n+j$ and $\left(g_{j}\right)_{k l}=0$ otherwise.

(i) If $I$ is a proper right ideal of $R$, then either there are subsets $\emptyset \neq X \subseteq n$ and $Y \subseteq p$ such that $I=\left(\bigoplus \sum_{i \in X} f_{i} R\right) \oplus\left(\bigoplus \sum_{j \in Y} g_{j} R\right)$ or $I \subseteq \operatorname{Soc}(R)$.

(ii) $\operatorname{Soc}(R)=\left\{\left(\begin{array}{cc}A & B \\ 0 & C\end{array}\right) \in R ; A=0\right\}$ and $\operatorname{Rad}(R)=\left\{\left(\begin{array}{cc}A & B \\ 0 & C\end{array}\right) \in R ; A=0, C=0\right\}$.

(iii) There is a ring isomorphism $R / \operatorname{Soc}(R) \cong M_{m}(T)$. 
(iv) $R$ is an indecomposable right hereditary right artinian ring. The set $\left\{f_{0}, g_{0}\right\}$ is a basic set of idempotents of $R$, and $\bar{R}=U T(m, S, T)$ is the basic ring of $R$.

(v) Put $P=g_{0} R$ and $J=f_{0} R / \operatorname{Soc}\left(f_{0} R\right)$. Then $\{P, J\}$ is a representative set of all simple modules. Moreover, $P$ is projective, but not injective, while $J$ is $\sum$-injective, but not projective.

(vi) $I(R)=M_{m \cdot n+p}(S)$, and the maximal right quotient ring of $R$ is isomorphic to $M_{m . n+p}(S)$.

Proof. By 5.3 and 5.6.

Another case when $R$ is of type II is that of $m=n=1$ :

Example 5.10. Let $0<p<\aleph_{0}$. Let $T$ and $S$ be skew-fields such that $T$ is a subring of $S$. Let $R=G T(1,1, p, S, T)$. Then $R$ is of type II.

Proof. Let $M$ be a cyclic non-projective module and let $N$ be a module such that $\operatorname{Ext}_{R}(M, N)=0$. We shall prove that $N$ is injective.

In view of 5.9(iv), w.l.o.g. we can assume that $M$ is indecomposable and that $M$ has a projective cover. By 5.9 (i) and (ii), this implies that $M \cong f_{0} R / \operatorname{Soc}\left(f_{0} R\right) \cong J$, where $\operatorname{Soc}\left(f_{0} R\right)=\operatorname{Rad}(R)$. Take $r \in Q=M_{1+p}(S)$ such that $r_{0 p}=1$ and $r_{k l}=0$ otherwise. Then $r R=\operatorname{Rad}(R)$. By 5.9(v), w.l.o.g. we can assume that the trace of $J$ in $N$ is 0 . Hence, there is a cardinal $\kappa>0$ such that

$$
\begin{aligned}
P^{(\kappa)} & \cong\left\{\left(\begin{array}{ll}
0 & d
\end{array}\right) ; d \in C F M_{\kappa \times p}(S)\right\}=\operatorname{Soc}(N) \subseteq N \subseteq I(N) \\
& =\left\{\left(d^{\prime} d\right) ; d^{\prime} \in C F M_{\kappa \times 1}(S), d \in C F M_{\kappa \times p}(S)\right\}=Q^{(\kappa)} .
\end{aligned}
$$

Take an arbitrary $a \in C F M_{\kappa \times 1}(S)$. Define $\varphi \in \operatorname{Hom}_{R}(\operatorname{Rad}(R), N)$ by $\varphi(r)=$ $(z a)$, where $z$ is the zero matrix in $C_{\kappa \times p}(S)$. Since $\operatorname{Ext}_{R}(M, N)=0, \varphi$ extends to some $\phi \in \operatorname{Hom}_{R}\left(f_{0} R, N\right)$, i.e. there is some $x \in N$ with $x f_{0}=x$ and $x r=\varphi(r)$. This implies that $x=(a z)$. Since $a$ was arbitrary, we infer that $N=I(N)$, i.e. $N$ is injective.

We are going to show that $R$ is not of type II in all remaining cases. First, we prove this in the case when $m>1$ :

Lemma 5.11. Let $0<n, p<\aleph_{0}$ and $1<m<\aleph_{0}$. Let $T$ and $S$ be skew-fields such that $T$ is a subring of $M_{m}(S)$. Let $R=G T(m, n, p, S, T)$. Then there is a non-projective cyclic module $M$ which is not i-test.

Proof. We shall use the notation of 5.9. Put $Q=M_{m . n+p}(S)$. Let $M=f_{0} R / r R$, where $r \in \operatorname{Rad}(R)$ is defined by $r_{0, m . n+p-1}=1$, and $r_{k l}=0$ otherwise. Since $r \in \operatorname{Rad}\left(f_{0} R\right)=\operatorname{Soc}\left(f_{0} R\right), M$ is not projective.

Further, $J=f_{0} R / \operatorname{Soc}\left(f_{0} R\right)$ is also a right $M_{n}(T)$-module of dimension 1. Denote by $C$ the set of all matrices $c \in f_{0} Q$ such that there exists $t \in T$ satisfying $c_{j 0}=t_{j 0}$ for all $j<m$, and $c_{k l}=0$ otherwise. Denote by $D$ the right $M_{n}(T)$ submodule of $f_{0} Q / \operatorname{Soc}\left(f_{0} R\right)$ generated by the cosets $c+\operatorname{Soc}\left(f_{0} R\right), c \in C$. By a matrix rank argument, it follows that $D \cap J=0$. So there is a maximal right $M_{n}(T)$ submodule, $E$, of $f_{0} Q / \operatorname{Soc}\left(f_{0} R\right)$ satisfying $D \subseteq E$ and $E \oplus J=f_{0} Q / \operatorname{Soc}\left(f_{0} R\right)$. Define a module $N$ by $\operatorname{Soc}\left(f_{0} R\right) \subseteq N \subset f_{0} Q, C \subseteq N$, and $N / \operatorname{Soc}\left(f_{0} R\right)=E$. Since $f_{0} Q$ is injective (see $5.9(\mathrm{vi})$ ) and $\operatorname{Soc}(N)=\operatorname{Soc}\left(f_{0} R\right)=\operatorname{Soc}\left(f_{0} Q\right) \triangleleft f_{0} Q$, we infer that $N$ is not injective. 
It remains to prove that $\operatorname{Ext}_{R}(M, N)=0$. Let $\varphi \in \operatorname{Hom}_{R}(r R, N)$. Then $\varphi(r)=$ ( $z$ a ) for some $a \in M_{m \times 1}(S)$, where $z$ is the zero matrix in $M_{m \times(m . n+p-1)}(S)$. Put $x=(a z) \in f_{0} Q$. Since $E \oplus J=f_{0} Q / \operatorname{Soc}\left(f_{0} R\right)$ and $\operatorname{Soc}\left(f_{0} R\right) \subseteq N$, there exist $b \in N$ and $s \in R$ such that $x=b+f_{0} s$. Moreover, since $C \subseteq N$, there is some $b^{\prime} \in N$ such that $b^{\prime} r=f_{0} s r$ and $b_{k l}^{\prime}=0$ for all $k<m$ and $0<l<m . n+p$. Define $\phi \in \operatorname{Hom}_{R}\left(f_{0} R, N\right)$ by $\phi\left(f_{0}\right)=b f_{0}+b^{\prime} f_{0}$. Then $\phi(r)=b r+f_{0} s r=x r=\varphi(r)$, i.e. $\phi$ extends $\varphi$. This proves that $\operatorname{Ext}_{R}(M, N)=0$.

We turn to the remaining case of $m=1, n>1$ and $T \neq K$. First, we need a lemma from non-commutative linear algebra:

Lemma 5.12. Let $T \subset S$ be skew-fields, and $1<n<\aleph_{0}$. Then there are a cardinal $\kappa$, a proper right $M_{n}(T)$-submodule $X$ of $C F M_{\kappa \times n}(S)$ and $y \in M_{n \times 1}(S)$ such that $X y=C F M_{\kappa \times 1}(S)$.

Proof. Put $\lambda=\operatorname{card}(T)$, i.e. $T=\left\{t_{\alpha} ; \alpha<\lambda\right\}$. Let $\left\{b_{\beta} ; \beta<\mu\right\}$ be a right $T$-basis of $S$ such that $b_{0}=1$. By the premise, $\mu>1$. Put $P=C F M_{(1+\lambda) \times 1}(S)$. For each $\alpha<\lambda$, define $x_{\alpha}, y_{\alpha} \in P$ by $\left(x_{\alpha}\right)_{00}=t_{\alpha},\left(x_{\alpha}\right)_{1+\alpha, 0}=b_{1}$ and $\left(x_{\alpha}\right)_{k l}=0$ otherwise, and by $\left(y_{\alpha}\right)_{1+\alpha, 0}=b_{0}$ and $\left(y_{\alpha}\right)_{k l}=0$ otherwise. Let $Y$ be a right $T$-subspace of $P$ generated by $\left\{x_{\alpha} ; \alpha<\lambda\right\} \cup\left\{y_{\alpha} ; \alpha<\lambda\right\}$. Define $p \in P$ by $p_{00}=1$ and $p_{k l}=0$ otherwise. Then $Y \cap p T=0$, i.e. there is a right $T$-subspace $Z \subset P$ such that $Z \oplus p T=P$ and $Y \subseteq Z$

Put $Q=C F M_{(1+\lambda) \times n}(S)$. Let $X=\left\{q \in Q ; \forall i<n \exists p \in Z \forall j<1+\lambda: q_{j i}=\right.$ $\left.p_{j 0}\right\}$. Clearly, $X$ is a proper right $M_{n}(T)$-submodule of $Q$. Define $y \in M_{n \times 1}(S)$ by $y_{00}=1, y_{10}=-b_{1}$, and $y_{k l}=0$ otherwise. Take $a \in P$. By construction, there exist $\alpha<\lambda$ and $z \in Z$ such that $a=z+p t_{\alpha}$. Define $x^{\prime} \in X$ so that the first column of $x^{\prime}$ is $x_{\alpha}$, the second is $y_{\alpha}$, and all other columns are zero. Then $x^{\prime} y=p t_{\alpha}$. Moreover, trivially, $z \in X y$. So $X y \supseteq Z \oplus p T=P$. Finally, it suffices to put $\kappa=\operatorname{card}(1+\lambda)$.

Lemma 5.13. Let $0<p<\aleph_{0}$ and $1<n<\aleph_{0}$. Let $T$ and $S$ be skew-fields such that $T$ is a proper subring of $S$. Let $R=G T(1, n, p, S, T)$. Then there is a non-projective cyclic module $M$ which is not $i$-test.

Proof. We shall use the notation of 5.9 and 5.12. Put $f=f_{0}+\cdots+f_{n-1}$ and $M=f R / r R$, where $r \in \operatorname{Rad}(R)$ is defined by $r_{i, n+p-1}=y_{i 0}$ for all $i<n$, and $r_{k l}=0$ otherwise. Since $r \in \operatorname{Rad}(f R), M$ is not projective.

Put $Q=C F M_{\kappa \times(n+p)}(S)$. Note that $\operatorname{Soc}(Q)$ consists of the $q \in Q$ such that the first $n$ columns of $q$ are zero. Put $N=\left\{q \in Q ; \exists x \in X: q_{\alpha i}=x_{\alpha i} \forall i<n \forall \alpha<\kappa\right\}$. Since $X$ is a proper subset of $C F M_{\kappa \times n}(S)$, we have $\operatorname{Soc}(Q)=\operatorname{Soc}(N) \unlhd N \triangleleft Q$. In particular, $N$ is not injective.

We prove that $\operatorname{Ext}_{R}(M, N)=0$. Let $\varphi \in \operatorname{Hom}_{R}(r R, N)$. Then $\varphi(r)=(z a)$ for some $a \in C F M_{\kappa \times 1}(S)$, where $z$ is the zero matrix in $C F M_{\kappa \times(n+p-1)}(S)$. By 5.12, there is some $x \in X$ with $x y=a$. Take $b \in N$ such that $b_{\alpha i}=x_{\alpha i}$ for all $\alpha<\kappa$ and $i<n$, and $b_{\alpha j}=0$ for all $\alpha<\kappa$ and $n \leq j<n+p$. Then $b f=b$, i.e. there is $\varphi \in H_{o m}(f R, N)$ such that $\varphi(f)=b$. Since $\varphi(r)=b r=x y=a, \phi$ extends $\varphi$. This means that $\operatorname{Ext}_{R}(M, N)=0$.

5.10 and 5.13 show that the property " $R$ is $\lambda$-saturated" is not Morita invariant for $\lambda=1$. This contrasts with the case of $\lambda \geq \aleph_{0}$ described in 5.7. 
Theorem 5.14. Let $R$ be a ring. Then $R$ is of type II if and only if there exist $0<n, p<\aleph_{0}$ and skew-fields $T \subseteq S$ such that $R$ is isomorphic to $G T(1, n, p, S, T)$, and either $T=S$ or $n=1$.

Proof. By 5.4, 5.6, 5.8, 5.10, 5.11 and 5.13.

We sum up our results for the case when $R$ is 1-saturated. To simplify notation, we shall write $R=R^{\prime}\left(\boxplus R^{\prime \prime}\right)$ to denote that either $R=R^{\prime}$ or $R=R^{\prime} \boxplus R^{\prime \prime}$ :

Theorem 5.15. Let $R$ be a 1-saturated ring. Then $R=R^{\prime}\left(\boxplus R^{\prime \prime}\right)$, where $R^{\prime \prime}$ is a semisimple ring and $R^{\prime}$ is an indecomposable 1-saturated ring. Moreover, either

(i) $R^{\prime}$ is of type Ia and $R^{\prime}$ is isomorphic to a full matrix ring over a local right artinian ring, or

(ii) $R^{\prime}$ is of type $I b$ and $R^{\prime}$ is a simple ring such that each right ideal is countably generated, or

(iii) $R^{\prime}$ is of type Ic and $R^{\prime}$ is right noetherian, with $\operatorname{Soc}\left(R^{\prime}\right)=0$,

or

(iv) $R^{\prime}$ is of type II, i.e. there exist $0<n, p<\aleph_{0}$ and skew-fields $T \subseteq S$ such that $R^{\prime}$ is isomorphic to $G T(1, n, p, S, T)$, and either $T=S$ or $n=1$.

Proof. By 4.9, 4.11 and 5.14.

Now, it is easy to see which of the rings of type II are 2-saturated:

Theorem 5.16. Let $R$ be of type II. Let $\kappa \geq 2$. Then $R$ is $\kappa$-saturated iff $R$ is Morita equivalent to $U T_{2}(S)$ for a skew-field $S$ iff $R \cong G T(1, n, p, S, S)$ for some $0<n, p<\aleph_{0}$ and a skew-field $S$.

Proof. Assume $R$ is of type II and $R$ is 2 -saturated. By 5.15 , we can assume that $R \cong G T(1, n, p, S, T)$ for some $0<n, p<\aleph_{0}$ and some skew-fields $T \subseteq S$ such that either $T=S$ or $n=1$.

Assume $n=1$ and $T \neq S$. Let $\bar{R}=M_{2}(R)$. Define $e \in \bar{R}$ by $e_{00}=1$ and $e_{k l}=0$ otherwise. Then $\bar{R}$ is Morita equivalent to $R$, the pair of adjoint functors realizing category equivalence between $\operatorname{Mod}-\bar{R}$ and $\operatorname{Mod}-R$ being $F=\operatorname{Hom}_{\bar{R}}(e \bar{R},-)$ and $G=-\otimes_{R} e \bar{R}$. Note that $\bar{R} \cong M_{2}(G T(1,1, p, S, T)) \cong G T(1,2,2 p, S, T)$. By 5.13 , there are a cyclic non-projective right $\bar{R}$-module $M$ and a non-injective right $\bar{R}$ module $N$ such that $\operatorname{Ext}_{\bar{R}}(M, N)=0$. By well-known properties of $F$, we have $\operatorname{Ext}_{R}(F(M), F(N))=0, F(M)$ is non-projective and $F(N)$ non-injective. Since $F(\bar{R}) \cong \bar{R} e \cong R^{(2)}$ in $\operatorname{Mod}-R, F(M)$ is 2-generated, and $R$ is not 2-saturated, a contradiction. This proves that $T=S$.

The remaining assertions follow from 5.8.

\section{ELIMINATION FOR TYPE I}

Though $\S 5$ provides a complete characterization of rings of type II, the case of type I is far from being solved. Nevertheless, applying various techniques of ring and module theory, we can "eliminate" this case, i.e. prove that there are no $\kappa$-saturated rings of type Ix provided $\kappa$ is "big enough".

This is easy to do for type Ic: by 4.3 and 4.11 (iii), no ring of type Ic is $\aleph_{0}$ saturated. For type Ib, we have a corresponding consistency result, Theorem 6.6. Moreover, by Theorem 6.1(ii) and Proposition 6.3, there are no $\aleph_{0}$-saturated rings of type Ib of cardinality $<2^{\aleph_{0}}$ (in ZFC). 
By 4.5, a complete elimination for type Ia is not possible. Nevertheless, using a generalization of Bongartz Lemma, we show that the description gets closer to 4.5 provided $\kappa$ is "big enough" (Theorems 6.11 and 6.13).

We start with the elimination for $\kappa=\aleph_{0}$ :

Theorem 6.1. Let $R$ be an $\aleph_{0}$-saturated ring. Then $R=R^{\prime}\left(\boxplus R^{\prime \prime}\right)$, where $R^{\prime \prime}$ is a semisimple ring and $R^{\prime}$ is an indecomposable $\aleph_{0}$-saturated ring. Moreover, either

(i) $R^{\prime}$ is of type Ia and $R^{\prime}$ is isomorphic to a full matrix ring over a local right artinian ring, or

(ii) $R^{\prime}$ is of type $I b$ and $R^{\prime}$ is a simple von Neumann regular ring such that all right ideals are countably generated and all simple right $R^{\prime}$-modules are isomorphic, or

(iii) $R^{\prime}$ is Morita equivalent to $U T_{2}(S)$ for a skew-field $S$.

Proof. By 4.3, 4.11(iii) and 5.16.

Corollary 6.2. Let $R$ be a right non-singular ring such that $R$ is not von Neumann regular. Then the following conditions are equivalent:

(i) $R$ is $\aleph_{0}$-saturated,

(ii) $R$ is fully saturated,

(iii) $\mathcal{P} \mathcal{T}$ is fully saturated,

(iv) $R=R^{\prime}\left(\boxplus R^{\prime \prime}\right)$, where $R^{\prime \prime}$ is a semisimple ring and there is a skew-field $S$ such that $R^{\prime}$ is Morita equivalent to $U T_{2}(S)$.

To eliminate type $\mathrm{Ib}$, we first show that there are no von Neumann regular rings of that type of size $<2^{\aleph_{0}}$ :

Proposition 6.3. Let $R$ be a simple von Neumann regular ring with card $(R)<$ $2^{\aleph_{0}}$. Then there are at least $2^{\aleph_{0}}$ non-isomorphic simple modules.

Proof. First, we define a 2-branching tree $(T,<)$ of height $\omega$ as follows: $T=$ $\bigcup_{n<\aleph_{0}} T_{n}$, where $T_{n}$ is the $n$-th level of $T$ and $T_{n}$ consists of a complete set of orthogonal idempotents of $R$ defined by induction as follows: $T_{0}=\{1\}$; if $e \in T_{n}$, then $R e R=R$, i.e. the rings $e R e$ and $R$ are Morita equivalent, whence there are orthogonal idempotents $f_{e}, g_{e} \in R$ such that $f_{e} \neq e \neq g_{e}$ and $e=f_{e}+g_{e}$, and we put $T_{n+1}=\bigcup_{e \in T_{n}}\left\{f_{e}, g_{e}\right\}$. Since $T_{n}$ is a complete set of idempotents, so is $T_{n+1}$. If $n<\aleph_{0}, e \in T_{n}$ and $e^{\prime} \in T_{n+1}$, we define $e \prec e^{\prime}$ iff either $e^{\prime}=f_{e}$ or $e^{\prime}=g_{e}$. Now, $<$ is defined as the transitive closure of $\prec$ on $T$. Denote by $B$ the set of all branches of $T$. Clearly, $\operatorname{card}(B)=2^{\aleph_{0}}$. For each $b \in B$, define a right ideal $I_{b}$ of $R$ by $I_{b}=\sum_{e \in(T \backslash b)} e R$. Then $I_{b} \neq R$, and there is a maximal right ideal $J_{b}$ of $R$ such that $I_{b} \subseteq J_{b}$.

Let $M$ be a simple module. Put $B_{M}=\left\{b \in B ; R / J_{b} \cong M\right\}$. Since $R$ is a simple ring, the Jacobson density theorem implies that we can view $R$ as a subring of $\operatorname{End}_{K}(M)$, where $K=\operatorname{End}_{R}(M)$ is a skew-field. Since $M$ is isomorphic to a factor module of $R, \operatorname{card}(M) \leq \operatorname{card}(R)<2^{\aleph_{0}}$ and $\operatorname{dim}_{K}(M) \leq \operatorname{card}(R) \ll 2^{\aleph_{0}}$. Let $b \in B_{M}$. Then $\operatorname{Hom}_{R}\left(R / J_{b}, M\right) \neq 0$ and there is some $0 \neq m_{b} \in M$ such that $m_{b} I_{b}=0$.

We shall prove that the set $U_{M}=\left\{m_{b} ; b \in B_{M}\right\}$ is a $K$-independent subset of $M$. On the contrary, let $\left\{m_{b_{i}} ; i<n\right\}$ be a $K$-dependent subset of $U_{b}$ having a minimal cardinality, $n>1$. Then $\sum_{i<n} k_{i} m_{b_{i}}=0$ for some $0 \neq k_{i}, i<n$. Since all the branches $b_{i}, i<n$, are different, there is some $e \in b_{0} \backslash \bigcup_{0<i<n} b_{i}$. Take $p<\aleph_{0}$ such that $e \in T_{p}$. Since $T_{p}$ is complete, $1-e$ is a sum of some elements 
of $T \backslash b_{0}$. Then $0=\sum_{i<n} k_{i} m_{b_{i}}(1-e)=\sum_{0<i<n} k_{i} m_{b_{i}}$, in contradiction with the minimality of $n$. Finally, denote by $\mathcal{S}$ a representative set of all simple modules. Clearly, $B=\bigcup_{M \in \mathcal{S}} B_{M}$. Since $\operatorname{card}\left(B_{M}\right) \leq \operatorname{dim}_{K}(M) \leq \operatorname{card}(R)<2^{\aleph_{0}}$ for each $M \in \mathcal{S}$, we infer that $\operatorname{card}(\mathcal{S}) \geq 2^{\aleph_{0}}$.

6.1(ii) and 6.3 now give

Corollary 6.4. Let $R$ be a right non-singular ring such that $\operatorname{card}(R)<2^{\aleph_{0}}$. Then the conditions (i)-(iv) of 6.2 are equivalent.

The remaining case of $\operatorname{card}(R) \geq 2^{\aleph_{0}}$ is covered by the following consistency result:

Lemma 6.5. Let $\kappa$ be a cardinal such that $\mathrm{cf}(\kappa)=\aleph_{0}$. Assume $\mathrm{UP}_{\kappa}$. Let $R$ be a non-right perfect ring such that $\operatorname{card}(R)<\kappa$. Then $\mathcal{P} \mathcal{T}$ is not 1-saturated, and $R$ is not $\kappa^{+}$-saturated.

Proof. Since $R$ is not semisimple, [Os, Corollary 2.23] implies that there exists a cyclic non-injective module, $N$. Clearly, $\operatorname{card}(N) \leq \operatorname{card}(R)<\kappa$. By 2.2 and 2.4, there is a non-projective module $M$ such that $\operatorname{gen}(M) \leq \kappa^{+}$and $\operatorname{Ext}_{R}(M, N)=0$. Hence, $N$ is not p-test, and $M$ is not i-test.

Thus, using UP, we reach elimination of types Ib and Ic in case $\kappa$ is "big enough":

Theorem 6.6. Assume UP. Let $R$ be a ring. Let $\kappa$ be a cardinal such that $\mathrm{cf}(\kappa)=$ $\aleph_{0}$ and $\operatorname{card}(R)<\kappa$. Assume $R$ is $\kappa^{+}$-saturated. Then $R=R^{\prime}\left(\boxplus R^{\prime \prime}\right)$, where $R^{\prime \prime}$ is a semisimple ring and either

(i) $R^{\prime}$ is isomorphic to a full matrix ring over a local right artinian ring, or

(ii) $R^{\prime}$ is Morita equivalent to $U T_{2}(S)$ for a skew-field $S$.

Proof. By 6.1 and 6.5.

UP also gives the following corollary (cf. 6.2 and 6.4):

Corollary 6.7. Assume UP. Let $R$ be a right non-singular ring. Then the following conditions are equivalent:

(i) $R$ is fully saturated,

(ii) $\mathcal{P} \mathcal{T}$ is fully saturated,

(iii) $R=R^{\prime}\left(\boxplus R^{\prime \prime}\right)$, where $R^{\prime \prime}$ is a semisimple ring and there is a skew-field $S$ such that $R^{\prime}$ is Morita equivalent to $U T_{2}(S)$.

By 4.5, artinian valuation rings provide examples of rings of type Ia. For further study of type Ia, we need a generalization to rings and modules of a well-known technique of Bongartz ([B, 2.1 Lemma] and [H, Lemma III.6.1]):

Lemma 6.8. Let $R$ be a ring and $A, B$ be modules. Assume $\operatorname{Ext}_{R}\left(B, B^{(\kappa)}\right)=0$ for all cardinals $\kappa$. Then there are a cardinal $\lambda$ and a module $C$ satisfying

(i) $\operatorname{Ext}_{R}(B, C)=0$, and

(ii) there is an exact sequence $0 \rightarrow A \rightarrow C \rightarrow B^{(\lambda)} \rightarrow 0$ in Mod-R.

Proof. Let $\lambda=\operatorname{gen}\left({ }_{\mathbb{Z}} E x t_{R}(B, A)\right)$. Take extensions

$$
0 \rightarrow A \rightarrow E_{\alpha} \rightarrow B \rightarrow 0, \quad \alpha<\lambda
$$


so that the equivalence classes of these extensions generate $\operatorname{Ext}_{R}(B, A)$ as an abelian group. Let

$$
0 \rightarrow A \rightarrow C \stackrel{\pi}{\longrightarrow} B^{(\lambda)} \rightarrow 0
$$

be the extension obtained by pushing out the direct sum extension

$$
0 \rightarrow A^{(\lambda)} \rightarrow \bigoplus \sum_{\alpha<\lambda} E_{\alpha} \rightarrow B^{(\lambda)} \rightarrow 0
$$

along $\nabla_{A} \in \operatorname{Hom}_{R}\left(A^{(\lambda)}, A\right)$ defined by $\nabla_{A}\left(\left(a_{\alpha} ; \alpha<\lambda\right)\right)=\sum_{\alpha<\lambda} a_{\alpha}$. Consider the long exact sequence

$$
\begin{gathered}
0 \rightarrow \operatorname{Hom}_{R}(B, A) \rightarrow \operatorname{Hom}_{R}(B, C) \rightarrow \operatorname{Hom}_{R}\left(B, B^{(\lambda)}\right) \stackrel{\delta}{\rightarrow} \operatorname{Ext}_{R}(B, A) \\
\rightarrow \operatorname{Ext}_{R}(B, C) \stackrel{\operatorname{Ext}_{R}(B, \pi)}{\longrightarrow} \operatorname{Ext}_{R}\left(B, B^{(\lambda)}\right)=0 \rightarrow \ldots
\end{gathered}
$$

induced by (2) and by the functors $\operatorname{Ext}_{R}^{i}(B,-)$. Since equivalence classes of the extensions (1) generate $\operatorname{Ext}_{R}(B, A)$, the connecting $\mathbb{Z}$-homomorphism $\delta$ is onto. Hence, the $\mathbb{Z}$-homomorphism $\operatorname{Ext}_{R}(B, \pi)$ is a monomorphism. This proves that $\operatorname{Ext}_{R}(B, C)=0$.

We shall also need a generalization of a bimodule dual of 6.8:

Lemma 6.9. Let $R$ and $S$ be rings, and let $A \in S-M o d-R$ and $B \in$ Mod-R. Put $\lambda=\operatorname{gen}\left({ }_{S} \operatorname{Ext}_{R}(B, A)\right)$. Assume $\operatorname{Ext}_{R}\left(A^{\lambda}, A\right)=0$. Then there is a module $C$ satisfying

(i) $\operatorname{Ext}_{R}(C, A)=0, \quad$ and

(ii) there is an exact sequence $0 \rightarrow A^{\lambda} \rightarrow C \rightarrow B \rightarrow 0$ in Mod-R.

Proof. Choose extensions

$$
0 \rightarrow A \rightarrow E_{\alpha} \stackrel{\rho_{\alpha}}{\longrightarrow} B \rightarrow 0, \quad \alpha<\lambda
$$

so that their equivalence classes generate $\operatorname{Ext}_{R}(B, A)$ as a left $S$-module. Let

$$
0 \rightarrow A^{\lambda} \stackrel{\mu}{\rightarrow} C \rightarrow B \rightarrow 0
$$

be the extension obtained by pulling back the direct product extension

$$
0 \rightarrow A^{\lambda} \rightarrow \prod_{\alpha<\lambda} E_{\alpha} \stackrel{\prod \rho_{\alpha}}{\longrightarrow} B^{\lambda} \rightarrow 0
$$

along $\Delta_{B} \in \operatorname{Hom}_{R}\left(B, B^{\lambda}\right)$ defined by $\Delta_{B}(b)=(b ; \alpha<\lambda)$. For each $\alpha<\lambda$, we have the following commutative diagram:

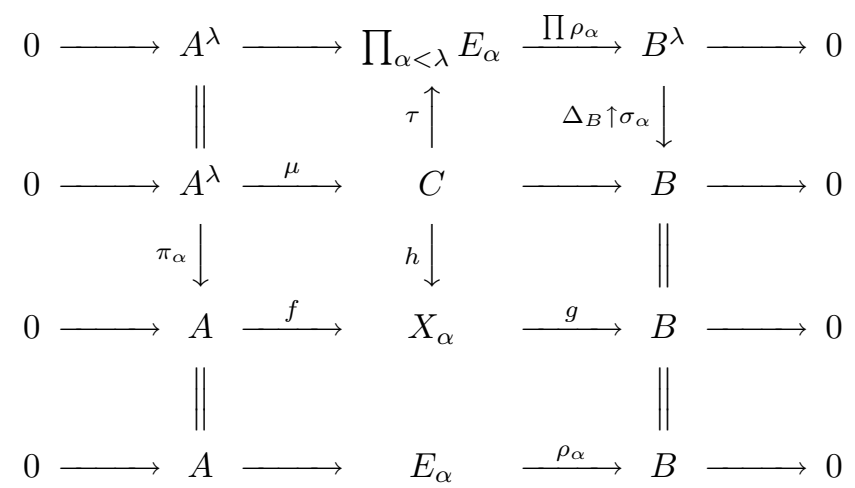


where $\sigma_{\alpha}$ is the $\alpha$-th projection of $B^{\lambda}$ to $B$, and the third row is obtained by pushing out the second row along the $\alpha$-th canonical projection, $\pi_{\alpha}$, of $A^{\lambda}$ onto $A$. Using the $\alpha$-th projection, $\eta_{\alpha}$, of $\prod_{\alpha<\lambda} E_{\alpha}$ onto $E_{\alpha}$, and the pushout property, we get $\varphi \in \operatorname{Hom}_{R}\left(X_{\alpha}, E_{\alpha}\right)$ making the lower left square commutative. Since $\operatorname{Im}(f)=\operatorname{Ker}(g), \operatorname{Im}(h)+\operatorname{Ker}(g)=X_{\alpha}$, and $g h=\sigma_{\alpha}\left(\prod \rho_{\alpha}\right) \tau=\rho_{\alpha} \eta_{\alpha} \tau=\rho_{\alpha} \varphi h$, we infer that also the lower right square is commutative. This means that the third and fourth rows are equivalent as extensions of $A$ by $B$. Consider the long exact sequence

$$
\begin{gathered}
0 \rightarrow \operatorname{Hom}_{R}(B, A) \rightarrow \operatorname{Hom}_{R}(C, A) \rightarrow \operatorname{Hom}_{R}\left(A^{\lambda}, A\right) \stackrel{\delta}{\rightarrow} \operatorname{Ext}_{R}(B, A) \\
\longrightarrow \operatorname{Ext}_{R}(C, A) \stackrel{\operatorname{Ext}_{R}(\mu, A)}{\longrightarrow} \operatorname{Ext}_{R}\left(A^{\lambda}, A\right)=0 \rightarrow \ldots
\end{gathered}
$$

induced by (4) and by the functors $\operatorname{Ext}_{R}^{i}(-, A)$. Since equivalence classes of the extensions (3) generate $\operatorname{Ext}_{R}(B, A)$, the commutative diagram constructed above shows that the connecting $S$-homomorphism $\delta$ is onto. Hence, the $S$-homomorphism $\operatorname{Ext}_{R}(\mu, A)$ is a monomorphism. This proves that $\operatorname{Ext}_{R}(C, A)=0$.

Now, we apply 6.8 and 6.9 to rings of type Ia:

Lemma 6.10. Let $R$ be a local right artinian ring. Assume $n=\operatorname{gen}\left({ }_{R} \operatorname{Soc}(R)\right)<$ $\aleph_{0}$ and $R$ is $(n+1)$-saturated. Then $n=1$ and $R$ is a QF-ring.

Proof. W.l.o.g, we assume that $R$ is not a skew-field. Then $\operatorname{Sing}(R) \neq 0$, and $R$ is not right hereditary. Take $0 \neq r \in \operatorname{Soc}(R)$. Denote by $\mu$ the inclusion of $r R$ into $R$. Since $R$ is local, there are left $R$-module isomorphisms

$$
\operatorname{Ext}_{R}(R / r R, R) \cong \operatorname{Hom}_{R}(r R, R) / \operatorname{Im}\left(\operatorname{Hom}_{R}(\mu, R)\right) \cong \operatorname{Soc}(R) / R r .
$$

In particular, $\lambda=\operatorname{gen}\left({ }_{R} \operatorname{Ext}_{R}(R / r R, R)\right) \leq n$. Put $A=R$ and $B=R / r R$. Since $R$ is local, $B$ is not projective. By 6.9 , we obtain a module $C$ such that $\operatorname{Ext}_{R}(C, R)=0$ and there is an exact sequence

$$
0 \rightarrow R^{\lambda} \rightarrow C \rightarrow B \rightarrow 0 .
$$

Proving indirectly, assume $C$ is projective. Since $R$ is not right hereditary, there is an exact sequence in $M o d-R$,

$$
0 \rightarrow N \rightarrow E \rightarrow E / N \rightarrow 0
$$

where $E$ is, but $E / N$ is not, injective. Applying $\operatorname{Ext}_{R}^{i}(-, N)$, we get

$$
0=\operatorname{Ext}_{R}\left(R^{\lambda}, N\right) \rightarrow \operatorname{Ext}_{R}^{2}(B, N) \rightarrow \operatorname{Ext}_{R}^{2}(C, N)=0
$$

and

$$
0=\operatorname{Ext}_{R}(B, E) \rightarrow \operatorname{Ext}_{R}(B, E / N) \rightarrow \operatorname{Ext}_{R}^{2}(B, N)=0 .
$$

This gives $\operatorname{Ext}_{R}(B, E / N)=0$. Since $R$ is 1-saturated, $B=R / r R$ is projective, a contradiction.

This proves that $C$ is not projective, but $\operatorname{Ext}_{R}(C, R)=0$. Since $\lambda \leq n$, (6) yields $\operatorname{gen}(C) \leq n+1$. Finally, $R$ is $(\mathrm{n}+1)$-saturated, so $R$ is injective and $R$ is a QF-ring by [AF, Theorem 30.7]. By (5), $R r=S o c(R)$ for each $0 \neq r \in S o c(R)$, i.e. $\operatorname{Soc}(R)$ is simple and $n=1$. 
Theorem 6.11. Let $R$ be a ring of type Ia. Assume $R$ is left artinian and $n$ saturated for all $n<\aleph_{0}$. Then $R$ is isomorphic to a full matrix ring over a local QF-ring.

Proof. By 4.11, $R \cong M_{m}(S)$ for some $0<m<\aleph_{0}$ and for a local left and right artinian ring $S$. By 5.7, $S$ satisfies the premises of 6.10 , whence $S$ and $R$ are QF-rings.

Lemma 6.12. Let $R$ be a right noetherian ring such that $R$ is not right hereditary. Assume $R$ is $\kappa$-saturated, for all $\kappa<\aleph_{0}$ and for all $\kappa \leq \operatorname{gen}(M)$, where $M$ is any indecomposable injective module. Then $R$ is a QF-ring.

Proof. Let $B$ be an indecomposable injective module. Since $R$ is right noetherian, we have $\operatorname{Ext}_{R}\left(B, B^{(\kappa)}\right)=0$ for all cardinals $\kappa$. Let $A$ be a non-injective module. By 6.8 , there are a cardinal $\lambda$ and a module $C$ such that $\operatorname{Ext}_{R}(B, C)=0$ and there is an extension

$$
0 \rightarrow A \rightarrow C \rightarrow B^{(\lambda)} \rightarrow 0
$$

Proving indirectly, assume $C$ is injective. Since $R$ is right noetherian, but not right hereditary, there is a non-projective finitely generated right ideal $I$ of $R$. Applying $\operatorname{Ext}_{R}^{i}(R / I,-)$ to the exact sequence (7), we get

$$
0=\operatorname{Ext}_{R}\left(R / I, B^{(\lambda)}\right) \rightarrow \operatorname{Ext}_{R}^{2}(R / I, A) \rightarrow \operatorname{Ext}_{R}^{2}(R / I, C)=0,
$$

whence $\operatorname{Ext}_{R}^{2}(R / I, A)=0$. Applying $\operatorname{Ext}_{R}^{i}(-, A)$ to the exact sequence $0 \rightarrow I \rightarrow$ $R \rightarrow R / I \rightarrow 0$, we get

$$
0=\operatorname{Ext}_{R}(R, A) \rightarrow \operatorname{Ext}_{R}(I, A) \rightarrow \operatorname{Ext}_{R}^{2}(R / I, A)=0 .
$$

Since $\operatorname{gen}(I)<\aleph_{0}, A$ is injective, a contradiction.

Thus, $C$ is not injective. Since $\operatorname{Ext}_{R}(B, C)=0$, our premise implies that $B$ is projective. This proves that any injective module is projective, and $R$ is a QF-ring by [AF, Theorem 31.9].

Theorem 6.13. Let $R$ be a ring of type Ia. Let $J$ be a representative of all simple modules, and put $\lambda=\operatorname{gen}(I(J))$. Assume $R$ is $\kappa$-saturated for all $\kappa<\max \left(\lambda^{+}, \aleph_{0}\right)$. Then $R$ is isomorphic to a full matrix ring over a local QF-ring.

Proof. By 4.11 and 6.12.

The following example shows that both $\operatorname{gen}\left({ }_{R} \operatorname{Soc}(R)\right)$ and $\operatorname{gen}(I(J))$ can be "arbitrarily" big for local right artinian rings which are not QF:

Example 6.14. Let $\lambda>1$ and let $1<n<\aleph_{0}$. Let $T, S$ be skew-fields such that $T$ is a subring of $S$, the left dimension of $S$ over $T$ is $\lambda$ and the right dimension is $n$ (see [Co] and [Sc]). Put $R=\left\{\left(\begin{array}{cc}t & s \\ 0 & t\end{array}\right) ; t \in T, s \in S\right\}$. Then

(i) $R$ is a subring of $M_{2}(S), R$ is local right artinian and $\operatorname{gen}\left({ }_{R} \operatorname{Soc}(R)\right)=\lambda$.

(ii) If $n=2$ and $\lambda \geq \aleph_{0}$, then $T$ and $S$ can be chosen so that $\operatorname{gen}(I(J))=\lambda$, where $J$ is a representative of all simple modules.

Proof. (i) Clearly, $\operatorname{Soc}(R)=\operatorname{Rad}(R)=\left\{\left(\begin{array}{cc}0 & s \\ 0 & 0\end{array}\right) ; s \in S\right\}$, whence $\operatorname{gen}\left({ }_{R} \operatorname{Soc}(R)\right)=\lambda$ and $\operatorname{gen}(\operatorname{Soc}(R))=n$. If $I \neq R$ is a left (right) ideal of $R$, then $I \subseteq \operatorname{Soc}(R)$. So $R$ is 
local and right artinian. Further, a module $N$ is injective iff $\operatorname{Ext}_{R}(R / \operatorname{Soc}(R), N)=$ 0 . This means that $R / \operatorname{Soc}(R)$ is an i-test module. Put $P=\left\{\left(\begin{array}{cc}s^{\prime} & s \\ 0 & 0\end{array}\right) ; s, s^{\prime} \in S\right\}$ and $L=\left\{\left(\begin{array}{ll}0 & t \\ 0 & 0\end{array}\right) ; t \in T\right\}$. Clearly, $L \cong J$. Moreover, $\operatorname{Ext}_{R}(R / \operatorname{Soc}(R), R) \neq 0$, $\operatorname{Ext}_{R}(R / \operatorname{Soc}(R), P) \neq 0$, while $\operatorname{Ext}_{R}(R / L, P)=0$. Thus, $R$ is not 1-saturated, and $R$ is not a QF-ring.

(ii) Assume $n=2$. Take $s \in S \backslash T$. Then $S=T \oplus s T$ in $M o d-T$. It follows that there are an injective ring homomorphism $\phi: T \rightarrow T$ and a $\phi$-differentiation $D: T \rightarrow T$ such that

$$
t s=s \phi(t)+D(t)
$$

for all $t \in T$ (see e.g. [Co, p.56]). This implies that the left dimension of $T$ over $\phi(T)$ is $\lambda-1$. Let $\left\{b_{\alpha} ; \alpha<\kappa\right\}$ be a right $\phi(T)$-basis of $T$ such that $b_{0}=1$. Put $L^{\prime}=\left\{\left(\begin{array}{cc}0 & s t \\ 0 & 0\end{array}\right) ; t \in T\right\}$. Denote by $\left\{e_{\alpha} ; \alpha<1+\kappa\right\}$ the canonical $R$-basis of the free module $R^{(1+\kappa)}$. Put

$$
K=e_{0} L^{\prime} \oplus\left(\bigoplus \sum_{\alpha<\kappa} e_{1+\alpha} L\right) \oplus\left(\bigoplus \sum_{\alpha<\kappa}\left(e_{0}\left(\begin{array}{ll}
0 & 1 \\
0 & 0
\end{array}\right)-e_{1+\alpha}\left(\begin{array}{cc}
0 & s b_{\alpha}^{-1} \\
0 & 0
\end{array}\right)\right) R\right)
$$

Let $M=R^{(1+\kappa)} / K$. For each $\alpha<1+\kappa$, let $m_{\alpha}=e_{\alpha}+K$. Clearly, $\left\{m_{\alpha} ; \alpha<\right.$ $1+\kappa\}$ is a generating subset of $M$. Moreover, $\operatorname{Soc}(M)=m_{0} L=m_{1+\alpha} L^{\prime} \cong J$, for all $\alpha<\kappa$, and $M / \operatorname{Soc}(M) \cong J^{(\kappa+1)}$. It follows that $\operatorname{gen}(M)=\kappa+1$, and $J \cong S o c(M) \unlhd M$. We shall prove that $M \cong I(J)$.

By (i), it suffices to show that $\operatorname{Ext}_{R}(R / \operatorname{Soc}(R), M)=0$. Put $r_{1}=\left(\begin{array}{ll}0 & 1 \\ 0 & 0\end{array}\right) \in$ $\operatorname{Soc}(R)$ and $r_{2}=\left(\begin{array}{cc}0 & s \\ 0 & 0\end{array}\right) \in \operatorname{Soc}(R)$. Then $\operatorname{Soc}(R)=r_{1} R \oplus r_{2} R$. Take any $\varphi \in$ $\operatorname{Hom}_{R}(\operatorname{Soc}(R), M)$, i.e. $\varphi\left(r_{1}\right)=m_{0}\left(\begin{array}{cc}0 & t_{1} \\ 0 & 0\end{array}\right)$ and $\varphi\left(r_{2}\right)=m_{0}\left(\begin{array}{cc}0 & t_{2} \\ 0 & 0\end{array}\right)$ for some $t_{1}, t_{2} \in$ $T$. Now, there exist $c_{\alpha} \in T, \alpha<\kappa$, such that $c_{\alpha}=0$ for almost all $\alpha<\kappa$, and $t_{2}-D\left(t_{1}\right)=\sum_{\alpha<\kappa} b_{\alpha} \phi\left(c_{\alpha}\right)$. Define $\psi \in \operatorname{Hom}_{R}(R, M)$ by

$$
\psi(1)=m_{0}\left(\begin{array}{cc}
t_{1} & 0 \\
0 & t_{1}
\end{array}\right)+\sum_{\alpha<\kappa} m_{1+\alpha}\left(\begin{array}{cc}
c_{\alpha} & 0 \\
0 & c_{\alpha}
\end{array}\right) .
$$

Then $\psi\left(r_{1}\right)=\psi(1) r_{1}=m_{0}\left(\begin{array}{cc}0 & t_{1} \\ 0 & 0\end{array}\right)=\varphi\left(r_{1}\right)$. By $(8)$,

$$
\begin{aligned}
\psi\left(r_{2}\right) & =\psi(1) r_{2}=m_{0}\left(\begin{array}{cc}
0 & D\left(t_{1}\right) \\
0 & 0
\end{array}\right)+\sum_{\alpha<\kappa} m_{1+\alpha}\left(\begin{array}{ccc}
0 & s \phi\left(c_{\alpha}\right) \\
0 & 0
\end{array}\right) \\
& =m_{0}\left(\begin{array}{cc}
0 & D\left(t_{1}\right) \\
0 & 0
\end{array}\right)+\sum_{\alpha<\kappa} m_{0}\left(\begin{array}{cc}
0 & b_{\alpha} \phi\left(c_{\alpha}\right) \\
0 & 0
\end{array}\right)=m_{0}\left(\begin{array}{cc}
0 & t_{2} \\
0 & 0
\end{array}\right)=\varphi\left(r_{2}\right) .
\end{aligned}
$$

This proves that $\psi \uparrow \operatorname{Soc}(R)=\varphi$, whence $\operatorname{Ext}_{R}(R / \operatorname{Soc}(R), M)=0$.

Finally, assume $\lambda \geq \aleph_{0}$. We choose $S$ and $T$ as in the Cohn construction [Co, pp. 124-126]. Then $\kappa=\operatorname{dim}\left(T_{\phi(T)}\right)=\operatorname{dim}\left({ }_{\phi(T)} T\right)=\lambda-1=\lambda$ (see [Co, pp. 125-126] or [CT1, Theorem 5.7]), and the assertion follows.

We sum up several results of Sections 5 and 6 : 
Theorem 6.15. Let $R$ be a fully saturated ring. Then $R=R^{\prime}\left(\boxplus R^{\prime \prime}\right)$, where $R^{\prime \prime}$ is a semisimple ring and $R^{\prime}$ is an indecomposable fully saturated ring. Moreover, either

(Ia) $R^{\prime}$ is isomorphic to a full matrix ring over a local QF-ring, or

(Ib) $R^{\prime}$ is a simple von Neumann regular ring such that all right ideals are countably generated and all simple right $R^{\prime}$-modules are isomorphic, or

(II) $R^{\prime}$ is Morita equivalent to $U T_{2}(S)$ for a skew-field $S$.

Proof. By 6.1 and 6.13 .

Theorem 6.16. Assume UP. Let $R$ be a fully saturated ring. Then $R=R^{\prime}\left(\boxplus R^{\prime \prime}\right)$, where $R^{\prime \prime}$ is a semisimple ring and either

(I) $R^{\prime}$ is of type Ia and $R^{\prime}$ isomorphic to a full matrix ring over a local QF-ring, or

(II) $R^{\prime}$ is of type II and $R^{\prime}$ is Morita equivalent to $U T_{2}(S)$ for a skew-field $S$.

Proof. By 6.6 and 6.15 .

\section{OPEN PROBLEMS}

(1) In 1.5, we have proved in $Z F C$ that $\mathcal{P} \mathcal{T}$ is a proper class for any right perfect ring $R$. If $R$ is not right perfect, then 2.5 shows that it is consistent with $Z F C$ that $\mathcal{P} \mathcal{T}$ is empty. On the other hand, if $R$ is right hereditary, then it is consistent with $Z F C$ that $\mathcal{P} \mathcal{T}$ is a proper class (see 3.13).

What is the possible size of $\mathcal{P} \mathcal{T}$ in the case when $R$ is non-right perfect and non-right hereditary ? Is then the assertion of 2.5 a theorem of ZFC ? The problem is open even in the very particular case of full endomorphism rings of infinite dimensional linear spaces over skew-fields (cf. 2.6 and 3.20).

(2) Is there a simple von Neumann regular ring $R$ such that $R$ is not semisimple and all simple modules are isomorphic (cf. 6.3, [OR] and [G2]) ?

(3) By 6.13, each fully saturated ring of type Ia is isomorphic to a full matrix ring over a local QF-ring $R$. Is $R$ actually an artinian valuation ring (as in 4.5) ?

\section{REFERENCES}

[AF] F.W.Anderson and K.R.Fuller, Rings and Categories of Modules, $2^{\text {nd }}$ edition, Springer, New York, 1992. MR 94i:16001

[As] I.Assem, Torsion theories induced by tilting modules, Canad.J.Math. 36 (1984), 899-913. MR 86j: 16022

[B] K.Bongartz, Tilted algebras, Representations of Algebras (Proc. Conf. Puebla, 1980), Springer, New York, 1981, pp. 26-38. MR 83j:16053

[CaEi] H.Cartan and S.Eilenberg, Homological Algebra, Princeton Univ.Press, Princeton, 1956. MR 17:1040e

[Co] P.M.Cohn, Skew Field Constructions, Cambridge Univ.Press, Cambridge, 1977. MR $57: 3190$

[CT1] R.Colpi and J.Trlifaj, Classes of generalized *-modules, Comm. Algebra 22 (1994), 39853995. MR 95e:16003

[CT2] R. Colpi and J. Trlifaj, Tilting modules and tilting torsion theories, J. Algebra (to appear).

[CMe] R. Colpi and C. Menini, On the structure of *-modules, J. Algebra 158 (1993), 400-419. MR 94i: 16003

[CzFa] J.H.Cozzens and C.Faith, Simple Noetherian Rings, Cambridge University Press, Cambridge, 1975. MR 53:522

[E] P.C.Eklof, Classification and non-classification results for abelian groups, Proc. Conf. "Abelian Groups and Modules", Padova 1994, Kluwer, Dordrecht, 1995 (to appear). 
[EM] P.C.Eklof and A.H.Mekler, Almost Free Modules, North-Holland, New York, 1990. MR 92e:20001

[ES1] P.C.Eklof and S.Shelah, On Whitehead modules, J. Algebra 142 (1991), 492-510. MR 92h:03077

[ES2] P.C.Eklof and S.Shelah, A combinatorial principle equivalent to the existence of nonfree Whitehead groups, Proc.Conf."Abelian Groups", Oberwolfach 1993, Contemporary Mathematics, vol. 171 Amer. Math. Soc., Providence, 1994, pp. 79-98. CMP 94:17

[Fa] C.Faith, Algebra: Rings, Modules and Categories I, Springer, New York, 1973. MR $\mathbf{5 1 : 3 2 0 6}$

[FuSa] L.Fuchs and L.Salce, Modules over Valuation Domains, M.Dekker, New York, 1985. MR 86h: 13008

[G1] K.R.Goodearl, Von Neumann Regular Rings, $2^{\text {nd }}$ edition, Krieger, Melbourne, 1991. MR 93m:16006

[G2] K.R.Goodearl, The Orsatti-Rodinò and Trlifaj counting methods for simple modules over regular rings.

[H] D.Happel, Triangulated Categories in the Representation Theory of Finite Dimensional Algebras, Cambridge University Press, Cambridge, 1988. MR 89e:16035

[Ho] W.Hodges, In singular cardinality, locally free algebras are free, Alg. Universalis 12 (1981) 205-220. MR 82i:08005

[K] L.Koifman, Rings over which each module has a maximal submodule, (in Russian), Mat. Zametki 3 (1970), 359-367. MR 41:6913

[L] J.Lambek, Lectures on Rings and Modules, Blaisdell, London, 1966. MR 34:5857

[N] R.J.Nunke, Modules of extensions over Dedekind rings, Illinois J. Math. 3 (1959), 222-241. MR 21:1329

[OR] A.Orsatti and N.Rodinò, On the endomorphism ring of an infinite dimensional vector space, Proc.Conf. "Abelian Groups and Modules", Padova 1994, Kluwer, Dordrecht, 1995 (to appear).

[Os] B.L.Osofsky, Homological Dimensions of Modules, C. B. M. S. Reg. Conf. Ser. in Math. 12, AMS, Providence, 1973. MR 56:5525

[Sc] A.H.Schofield, Representations of Rings over Skew Fields, Cambridge University Press, Cambridge, 1985. MR 87c:16001

[S1] S.Shelah, Infinite abelian groups, Whitehead problem and some constructions, Israel J. Math. 18 (1974), 243-256. MR 50:9582

[S2] S.Shelah, A compactness theorem for singular cardinals, free algebras, Whitehead problem and transversals, Israel J. Math. 21 (1975), 319-349. MR 52:10410

[S3] S.Shelah, Whitehead groups may not be free even assuming CH, I, Israel J. Math. 28 (1977), 193-203. MR 57:9538

[S4] S.Shelah, Whitehead groups may not be free even assuming CH, II, Israel J. Math. 35 (1980), 257-285. MR 82h:03055

[S5] S.Shelah, Proper Forcing, Springer, New York, 1982. MR 84h:03002

[Si] D.Simson, An Artin problem for division ring extensions and the pure semisimplicity conjecture (to appear).

[Sm] S.O.Smalø, Torsion theories and tilting modules, Bull.London Math.Soc. 16 (1984), 518522. MR 85k: 16038

[T1] J.Trlifaj, Associative Rings and the Whitehead Property of Modules, R. Fischer, Munich, 1990. MR 91h:16013

[T2] J.Trlifaj, Non-perfect rings and a theorem of Eklof and Shelah, Comment. Math. Univ. Carolinae 32 (1991), 27-32. MR 92h:03078

[T3] J.Trlifaj, Almost *-modules need not be finitely generated, Comm. Algebra 21 (1993), 2453-2462. MR 94e:16014

[T4] J.Trlifaj, Every *-module is finitely generated, J. Algebra 169 (1994), 392-398. CMP 95:02

Department of Algebra, Faculty of Mathematics and Physics, Charles University, Sokolovská 83, 18600 Prague 8, The Czech Republic

E-mail address: trlifaj@karlin.mff.cuni.cz 